Manuscript accepted in Neurobiology of Aging on September $14^{\text {th }}, 2019$

\title{
What Electrophysiology Tells Us About Alzheimer's Disease: A Window into the Synchronization and Connectivity of Brain Neurons
}

Claudio Babiloni ${ }^{1,2}$, Katarzyna Blinowska ${ }^{3}$, Laura Bonanni ${ }^{4}$, Andrej Cichocki $i^{5,6,7}$, Willem De Haan ${ }^{8}$, Claudio del Percio ${ }^{1}$, Bruno Dubois ${ }^{9,10}$, Javier Escudero ${ }^{11}$, Alberto Fernández ${ }^{12}$, Giovanni Frisoni ${ }^{13,14}$, Bahar Guntekin ${ }^{15}$, Mihaly Hajos ${ }^{16}$, Harald Hampel ${ }^{9,10,17}$, Emmanuel Ifeachor ${ }^{18}$, Kerry Kilborn ${ }^{19}$, Sanjeev Kumar ${ }^{20}$, Kristinn Johnsen ${ }^{21}$, Magnus Johannsson ${ }^{21}$, Jaeseung Jeong ${ }^{22}$, Fiona LeBeau ${ }^{23}$, Roberta Lizio ${ }^{24}$, Fernando Lopes da Silva ${ }^{25}$, Fernando Maestú12, William J. McGeown ${ }^{26}$, lan McKeith $^{23}$, Davide Vito Moretti ${ }^{13}$, Flavio Nobili27,28, John Olichney ${ }^{29}$, Marco Onofrj4, Jorge J. Palop ${ }^{30}$, Michael Rowan ${ }^{31}$, Fabrizio Stocchi32, Zbigniew Struzik ${ }^{33}$, Heikki Tanila34, Stefan Teipel ${ }^{35,36}$, John Paul Taylor ${ }^{23}$, Marco Weiergräber ${ }^{37}$, Gorsev Yener $^{38}$, Tracy Young-Pearse ${ }^{39}$, Wilhelmus H. Drinkenburg ${ }^{40}$, Fiona Randall ${ }^{41}$

1) Department of Physiology and Pharmacology "Vittorio Erspamer", Sapienza University of Rome, Rome, Italy;

2) Institute for Research and Medical Care, Hospital San Raffaele of Cassino, Cassino (FR), Italy;

3) Faculty of Physics University of Warsaw and Nalecz Institute of Biocybernetics, Warsaw, Poland;

4) Department of Neuroscience Imaging and Clinical Sciences and CESI, University G D'Annunzio of Chieti-Pescara, Chieti, Italy;

5) Skolkowo Institute of Science and Technology (SKOLTECH), Moscow, Russia;

6) Systems Research Institute PAS, Warsaw, Poland;

7) Nicolaus Copernicus University (UMK), Torun, Poland

8) Amsterdam Neuroscience, Department of Neurology, Amsterdam UMC, Vrije Universiteit Amsterdam, Amsterdam, The Netherlands;

9) Institute of Memory and Alzheimer's Disease (IM2A), Department of Neurology, Pitié-Salpêtrière Hospital, AP-HP, Boulevard de l'hôpital, Paris, France;

10) Institut du Cerveau et de la Moelle épinière, ICM, INSERM U1127, CNRS UMR 7225, Sorbonne Université, Paris, France;

11) School of Engineering, Institute for Digital Communications, The University of Edinburgh, Edinburgh, UK;

12) Laboratory of Cognitive and Computational Neuroscience, Center for Biomedical Technology, Universidad Complutense and Universidad Politécnica de Madrid, Madrid, Spain;

13) IRCCS San Giovanni di Dio Fatebenefratelli, Brescia, Italy;

14) Memory Clinic and LANVIE - Laboratory of Neuroimaging of Aging, University Hospitals and University of Geneva, Geneva, Switzerland;

15) Department of Biophysics, International School of Medicine, Istanbul Medipol University, Istanbul, Turkey

16) Translational Neuropharmacology, Department of Comparative Medicine, Yale University School of Medicine, New Haven, CT, USA;

17) $G R C n^{\circ} 21$, Alzheimer Precision Medicine (APM), AP-HP, Pitié-Salpêtrière Hospital, Boulevard de l'hôpital, Sorbonne University, Paris, France;

18) School of Computing, Electronics and Mathematics, Faculty of Science and Engineering, Plymouth University, Plymouth, UK;

19) School of Psychology, University of Glasgow, Glasgow, UK;

20) Geriatric Psychiatry Division, Centre for Addiction and Mental Health, Toronto, ON, Canada;

21) Mentis Cura ehf., Reykjavík, Iceland;

22) Department of Bio and Brain Engineering/Program of Brain and Cognitive Engineering Korea Advanced Institute of Science and Technology (KAIST), Daejeon, South Korea;

23) Institute for Neuroscience, Newcastle University, Newcastle upon Tyne, UK

24) IRCCS SDN, Napoli, Italy

25) Center of Neurosciences, Swammerdam Institute of Life Sciences, University of Amsterdam, Amsterdam, The Netherlands;

26) School of Psychological Sciences and Health, University of Strathclyde, Glasgow, UK;

27) Department of Neuroscience (DINOGMI), University of Genoa, Genoa, Italy;

28) Clinica Neurologica, IRCCS Ospedale Policlinico San Martino, Genoa, Italy;

29) UC Davis Department of Neurology and Center for Neuroscience, Davis, CA, USA,

30) Gladstone Institute of Neurological Disease, San Francisco, CA, USA

31) Department of Pharmacology and Therapeutics, Watts Building, Trinity College Dublin, Dublin, Ireland;

32) IRCCS San Raffaele Pisana, Rome, Italy;

33) RIKEN Advanced Center for Computing and Communication, Wako, Japan

34) A. I. Virtanen Institute, University of Eastern Finland, Kuopio, Finland

35) German Center for Neurodegenerative Diseases (DZNE), Rostock, Germany;

36) Department of Psychosomatic and Psychotherapeutic Medicine, University of Rostock, Rostock, Germany;

37) Experimental Neuropsychopharmacology, Federal Institute for Drugs and Medical Devices (Bundesinstitut für Arzneimittel und Medizinprodukte, BfArM), Bonn, Germany;

38) Department of Neurosciences and Department of Neurology, Dokuz Eylül University Medical School, Izmir, Turkey;

39) Ann Romney Center for Neurologic Diseases, Harvard Medical School and Brigham and Women's Hospital, Boston, Massachusetts, USA;

40) Janssen Research \& Development, Janssen Pharmaceutica NV, Beerse, Belgium;

41) Vertex Pharmaceuticals Incorporated, Boston, MA, USA.

Dr. Pim Drinkenburg and Dr. Fiona Randall equally contributed to this paper. 


\section{Conflicts of Interest}

Dr. Harald Hampel serves as Senior Associate Editor for the Journal Alzheimer's \& Dementia; he received lecture fees from Biogen and Roche, research grants from Pfizer, Avid, and MSD Avenir (paid to the institution), travel funding from Functional Neuromodulation, Axovant, Eli Lilly and company, Takeda and Zinfandel, GEHealthcare and Oryzon Genomics, consultancy fees from Jung Diagnostics, Cytox Ltd., Axovant, Anavex, Takeda and Zinfandel, GE Healthcare, Oryzon Genomics, and Functional Neuromodulation, and participated in scientific advisory boards of Functional Neuromodulation, Axovant, Eli Lilly and company, Cytox Ltd., GE Healthcare, Takeda and Zinfandel, Oryzon Genomics and Roche Diagnostics.

\section{Corresponding author:}

Prof. Claudio Babiloni, Ph.D.

Department of Physiology and Pharmacology "V. Erspamer"

Sapienza University of Rome

P. le A. Moro 5, 00185, Rome, Italy

Phone: +39 0649910989; E-mail: claudio.babiloni@uniroma1.it

\section{Acknowledgements}

This Position Paper is endorsed by the Electrophysiology Professional Interest Area (EPIA) of the Alzheimer's Association International Society to Advance Alzheimer's Research and Treatment (ISTAART).

The Authors thank the Executive Steering Committee of EPIA for their commitment to the production of this manuscript.

Dr. Fernando Lopes da Silva passed away due to health problems (Junuary $24^{\text {th }}, 1935-$ May $\left.7^{\text {th }}, 2019\right)$. Until the end of his life, he had been working on the development of EEG science and its application in Clinical Neurophysiology. He leaves to all us an invaluable scientific legacy in the Science of Brain Rhythms and Human Cognition. He also leaves a permanent reminder of scientific passion, academic soul, and social responsibility to all fortunate people who met and knew him during his intense life.

Dr. Claudio Babiloni, Dr. Stefan Teipel, and Dr. Emmanuel Ifeachor are supported by European Committee (H2020-EU.1.3.1.H2020-MSCA-ITN-ETN-2016 project with short title "BBDiag").

Dr. Fiona Randall is a full-time employee of 41) Vertex Pharmaceuticals Incorporated.

Dr. Pim Drinkenburg is a full-time employee and holds shares/options of Janssen Pharmaceutica NV, Pharmaceutical Companies of Johnson \& Johnson.

Dr. Harald Hampel is supported by the AXA Research Fund, the "Fondation partenariale Sorbonne Université" and the "Fondation pour la Recherche sur Alzheimer", Paris, France. Ce travail a bénéficié d'une aide de l'Etat "Investissements d'avenir" ANR-10-IAIHU-06. The research leading to these results has received funding from the program "Investissements d'avenir" ANR-10-IAIHU-06 (Agence Nationale de la Recherche-10-IA Agence Institut Hospitalo-Universitaire-6).

This publication benefited from the support of the Program "PHOENIX" led by the Sorbonne University Foundation and sponsored by la Fondation pour la Recherche sur Alzheimer.

Dr Fabrizio Stocchi and Dr. Roberta Lizio gave their contribution to this paper with the support of "Ricerca Corrente" funds of Italian Ministry of Health for the IRCCS "San Raffaele Pisana" of Rome and the IRCCS "SDN" of Naples, respectively.

\section{Key Words}

The Alzheimer's Association International Society to Advance Alzheimer's Research and Treatment (ISTAART); Alzheimer's disease (AD); Electroencephalography and Magnetoencephalography (EEG and MEG); Resting State Condition; Event-Related Potentials and Magnetic Fields. Preclinical and clinical research.

\section{Running Title:}

Electrophysiology in Alzheimer's disease research.

\section{Manuscript requirements:}

\begin{tabular}{lr}
\hline Title: & 1 s spaces \\
Abstract: & 143 words \\
Text: & 6,724 \\
References: & 135 \\
Table (Supp. material) & 1 \\
Panels & 4 \\
Figures & 3
\end{tabular}




\title{
HIGHLIGHTS
}

- Electrophysiology probes Alzheimer's effects on neurons/networks

- It also probes drug effects on neurons/networks

- Electrophysiology biomarkers measure channelopathy and synaptic neurotransmission

- They also measure neural network dynamics, synchronization, and connectivity

- $\quad$ Spatial scale ranges from microscopic $(\mu \mathrm{m})$ to macroscopic $(\mathrm{cm})$ measures

- Temporal scale ranges from microseconds to hours (e.g., sleep)

- Electrophysiology markers translate from clinical to preclinical research

\begin{abstract}
Electrophysiology provides a real-time readout of neural functions and network capability in different brain states, on temporal (fractions of milliseconds) and spatial (micro, meso, and macro) scales unmet by other methodologies. However, current international guidelines do not endorse the use of electroencephalographic (EEG)/magnetoencephalographic (MEG) biomarkers in clinical trials performed in patients with Alzheimer's disease (AD), despite a surge in recent validated evidence. This Position Paper of the ISTAART Electrophysiology Professional Interest Area endorses consolidated and translational electrophysiological techniques applied to both experimental animal models of $\mathrm{AD}$ and patients, to probe the effects of $\mathrm{AD}$ neuropathology (i.e., brain amyloidosis, tauopathy, and neurodegeneration) on neurophysiological mechanisms underpinning neural excitation/inhibition and neurotransmission as well as brain network dynamics, synchronization, and functional connectivity reflecting thalamocortical and cortico-cortical residual capacity. Converging evidence shows relationships between abnormalities in EEG/MEG markers and cognitive deficits in groups of AD patients at different disease stages. The supporting evidence for the application of electrophysiology in AD clinical research as well as drug discovery pathways warrants an international initiative to include the use of EEG/MEG biomarkers in the main multicentric projects planned in AD patients, to produce conclusive findings challenging the present regulatory requirements and guidelines for AD studies.
\end{abstract}




\section{INTRODUCTION}

The International Working Group (IWG) and the US National Institute on AgingAlzheimer's Association (NIA-AA) have recently proposed and refined guidelines for the diagnosis and monitoring of Alzheimer's disease (AD) at the preclinical, prodromal (with objective mild cognitive impairment (MCI) but with aut onomy substantially preserved), and overt dementia stages, based on in-vivo fluid and neuroimaging biomarkers (Dubois et al., 2014; Jack et al., 2018). According to these diagnostic guidelines, AD status is associated with (1) a reduction of cerebrospinal (CSF) A $\beta 42$ and an increase in amyloid at the brain level as revealed by amyloid positron emission tomography (PET) mapping, and (2) an increase of phospho-tau in both CSF and tau PET mapping. Neurodegeneration may be revealed by Fluorodeoxyglucose PET (FDG-PET), total tau in CSF, and magnetic resonance imaging (MRI) of brain atrophy in temporoparietal cortex and the hippocampi.

Whilst these methodologies capture relevant AD processes, their uses are limited in the general clinical setting due to cost and invasiveness, especially for serial recordings over time (Dubois et al., 2014). In this respect, electrophysiology offers a promising and practical alternative that is both non-invasive and cost-effective. Electrophysiology allows the investigation of neurophysiological mechanisms underlying neural ionic current flows and related voltages at different spatial scales in the brain at an insuperable time resolution (i.e. $<1 \mathrm{~ms})$.

Due to the huge amount of information embedded in the waves recorded as electrophysiological signals, and in the gray zone between signal and biological/instrumental noise, there are some uncertainties about how to choose the optimum electrophysiological markers to extract from the recorded signals for preclinical and clinical research in AD. Furthermore, there may be difficulties in choosing which of the markers to use in a study, because often several alternative electrophysiological markers could be informative for the same research purpose. To overcome these uncertainties, several independent experts of cellular neurophysiology, translational and clinical neurophysiology, pharmacoelectroencephalography (EEG), magnetoencephalography (MEG), mathematical modeling of complex phenomena, and AD biomarkers wrote the present White Paper to define approaches to maximize the potential of electrophysiology applications in AD research to inform both research, diagnostics, patient stratification, disease progression and clinical development. This Paper reports their cross-disciplinary, unanimous consensus and recommendations about 
evidence-based opportunities and limitationss offered by several modern electrophysiological markers for preclinical and clinical AD research.

\section{ELECTROPHYSIOLOGY AND ALZHEIMER'S DISEASE (AD)}

\subsection{Electrophysiology probes effects of Alzheimer's disease (AD) on brain neural synchronization and functional connectivity at different spatial scales}

Electrophysiology can be applied to investigate effects of $\mathrm{AD}$ processes (i.e. amyloidosis and tauopathy) on two main neurophysiological mechanisms underpinning human sensorimotor and cognitive functions: (1) synchronization/desynchronization of neural activities (i.e., post-synaptic and action potentials), as revealed by electrophysiological signals recorded in two or more neurons at a given frequency and location, and (2) coupling/decoupling of neural activities, as revealed by electrophysiological signals recorded in two or more populations of neurons.

These mechanisms can be investigated using electrophysiological techniques at different spatial scales providing extensive opportunity for integration and interpretation of translational information from experimental models to human subjects: (1) microscale $(<50$ $\mu \mathrm{m})$ for synaptic connections between pairs of neurons. Patch clamp is used to record ionic currents through the neural membrane while single unit recordings probe action and postsynaptic potentials. Experimental challenges can include several amyloid and tau species and therapeutic candidates; (2) mesoscale $(100 \mu \mathrm{m} r>1 \mathrm{~mm})$ for connections within and between circumscribed neural populations such as those organized within cortical minicolumns $(50 \mu \mathrm{m})$ and macrocolumns $(<1 \mathrm{~mm})$. Intracerebral recordings reveal local field potentials (LFPs) in animal AD models; and (3) macroscale (> $1 \mathrm{~mm}$ ) for connections between large neural populations as those organized within brain structures and regions. Intracerebral and epidural recordings collect LFPs and EEG activity in animal AD models, while extracranial recordings of EEG or MEG activity are collected in AD patients at different stages of the neuropathology. Figure 1 illustrates the spatial and temporal resolutions of the main electrophysiological techniques applied to preclinical and clinical AD research.

LFPs and EEG/MEG activity differ in relation to the generating mechanisms. LFPs are produced by the synchronization of activity and generation of post-synaptic potentials in several squared millimeters of brain pyramidal neurons (Nunez, 2000). EEG/MEG activity is generated by the synchronization of activity and generation of post-synaptic potentials in 
several squared centimeters of cortical pyramidal neurons. Of note, this synchronization is the output of the brain system generating LFPs and EEG/MEG activity (Nunez, 2000). Figure 2 illustrates how the main electrophysiological techniques can probe brain neural synchronization and coupling in vitro and in vivo.

General topological features of the functional connectivity between nodes of brain system generating LFPs and EEG/MEG activity can be explored by graph theory (Bullmore $\&$ Sporns, 2009). Some graph theory indices are information circulation, network robustness and adaptation to pathological disturbances, modularity and functional specialization of subnetworks, and "small-worldness" defined as a good balance between an intense local connectivity and effective "hubs" for the remote connectivity (Bullmore \& Sporns, 2009). This approach may be quite relevant for characterizing $\mathrm{AD}$ as a "brain disconnection syndrome" (van Straaten \& Stam, 2013). However, it still lacks an international consensus on several important procedural aspects such as spatial sampling of EEG/MEG activity, edge definition in connectivity, statistical thresholds for the determination of the existence of a connection, and how to deal with instrumental and/or biological noise in the data. These aspects should be well defined in future AD research (Vecchio et al., 2017; van Diessen et al., 2015; van Diessen et al., 2015; Smith and Escudero, 2017).

\subsection{Main features of electrophysiological signals}

Electrophysiological signals reveal unique neurophysiological dimensions of brain capacity and reserve in AD. These signals can be considered as reflections of outputs of neural systems regulating synchronization/desynchronization and functional coupling/decoupling in neural brain populations regulating vigilance, motivation, and cognitive processes (Nunez, 2000; Nunez et al., 2015; Voytek and Knight, 2015). Inputs to these systems derive from several interdependent neural circuits including (1) ascending activating fibers from brainstem and basal forebrain; (2) thalamocortical, corticothalamic, and cortical local interneurons; and (3) cortico-cortical fibers ensuring longitudinal intra-hemispheric and inter-hemispherical (e.g., corpus callosum, commissures, etc.) neural transmissions (Nunez, 2000; Fields, 2008; Voytek and Knight, 2015). These circuits are important components of the subcortical white matter and convey electrochemical oscillatory signals that regulate functional connectivity and synchrony in the brain neural spiking activity (Fields, 2008; Nunez et al., 2015). Indeed, myelinization of ascending fibers affects the speed of action potentials across axons and, then, the synchrony of volleys of action potentials, neurotransmitter release, and postsynaptic 
potentials at synapses within distributed cognitive systems underpinning mental operations, encoding-retrieval processes, learning, and executive functions (Nunez and Srinivasan, 2006; Nunez et al, 2015). Consequently, alterations in axon myelination and white matter integrity are associated with abnormalities in rsEEG rhythms and clinical manifestations (Voytek and Knight, 2015). For example, alterations in brain white matter and functional connectivity have been associated with abnormal rsEEG rhythms and cognitive processes in patients with schizophrenia, depression, Parkinson's disease, and other pathological conditions (Fields, 2008; Nunez et al., 2015; Voytek and Knight, 2015).

The relationships between these outputs and inputs of neural systems can be represented by values in the space of deterministic-random (i.e., stochastic), complexity-simplicity, linearnonlinear, stationary-nonstationary, and phase-nonphase locking dimensions that may be differently affected by AD processes. These dimensions may be candidate biomarkers of AD. However, their neurophysiological interpretation should always consider the following confounding factors: (1) head volume conduction through the brain, skull and scalp, of a myriad of extracellular ionic currents and associated post-synaptic and action potentials generated by neural interactions for billions of neurons and hundreds of brain regions. This head volume conduction produces not only the summation of extracellular ionic currents and associated potentials, but also their partial or complete cancellation when they are opposite in the polarity; (2) biological noise produced by non-neuronal ionic currents or potentials (e.g., ocular, muscle, and cardiac activities); and (3) instrumental noise due to recording systems and environmental electromagnetic sources. See the Panel 1 for more details.

\section{CLINICAL ELECTROPHYSIOLOGY OF AD}

Electrophysiology can be investigated in AD patients during a variety of experimental conditions including; sleep, quiet wakefulness, and during sensorimotor and cognitive events, and measurements can include spontaneous or triggered electrical activities permitting both investigation of general network activity and network responsiveness.

When endogenous or exogenous events trigger sensorimotor and cognitive information processing, event-related and phase-locked changes of this EEG (MEG) activity can be investigated by two linear procedures: (1) event-related potentials (ERPs) or their magnetic counterpart (event-related magnetic fields, ERMFs) and (2) event-related oscillations (EROs) or their magnetic counterpart (event-related magnetic oscillations, ERMOs). A typical ERP paradigm for $\mathrm{AD}$ research is the "auditory oddball task" in which patients receive a sequence 
of frequent ( $80 \%$ of probability) and rare (20\%) stimuli with the request to respond with a hand movement or silent counting only to the rare ones (for a review see Rossini et al., 2006).

Event-related and nonphase-locked changes of ongoing EEG (MEG) rhythms can be investigated by a linear procedure to produce the so-called event-related desynchronization/synchronization (ERD/ERS). More details on these paradigms are reported in Panel 2. Figure 3 illustrates the neurophysiological mechanisms at the basis of EEG/MEG generation.

\subsection{Resting state EEG/MEG rhythms in $\mathrm{AD}$ at the group level}

\section{EEG}

Amplitude or source activity of resting state eyes-closed cortical EEG rhythms probes the mechanisms of synchronization/desynchronization of thalamocortical and ascending activity systems in the maintenance of low vigilance in quiet wakefulness and can unveil the progressive effects of $\mathrm{AD}$ and intervention on these systems at the group level, even at late disease stages (direct assessment of the patient's cognitive skills is not required).

As a reference, cognitively normal old (Nold) subjects show prominent amplitude or source activity of resting state cortical EEG (MEG) rhythms at alpha frequencies (8-12 $\mathrm{Hz})$ in posterior regions and low amplitude of spatially widespread delta $(<4 \mathrm{~Hz})$ and theta (4-7 Hz) rhythms. Compared to Nold subjects, Alzheimer's Disease with Dementia (ADD) groups typically exhibit an increase of delta/theta rhythms and a decrease of alpha and beta (14-30 Hz) rhythms (Jelic et al., 2000; Brassen and Adler, 2003; Onofrj et al., 2003; Jeong, 2004). Compared with dementia due to Parkinson's Disease Dementia (PDD) and Lewy Body diseases (LBD), ADD groups are characterized by abnormally lower posterior alpha and less disruption of delta rhythms (Babiloni et al., 2017a).

Interrelatedness (e.g., spectral coherence) of EEG (MEG) rhythms at different scalp sensors or cortical sources probes the progressive effects of ADD and intervention on the mechanisms of coupling/decoupling of synchronization in thalamocortical and cortical neural activity. This approach is insightful as cognition is strictly related to synchronous patterns of activation in brain neural networks, regulated by neurophysiological oscillatory mechanisms (e.g., visual, spatial, somatosensory, and semantic contents of our mental images or thoughts). However, two caveats should be emphasized in the interpretation of the results. Computing the interrelatedness of EEG (MEG) cortical sources, using estimates of functional cortical connectivity in the maintenance of low vigilance, involves some 
mathematical assumptions, so different solutions correspond to different assumptions. Furthermore, whether interrelatedness (e.g., spectral coherence) of EEG (MEG) rhythms between EEG sensors can reliably reflect that functional cortical connectivity is an open issue.

Clinical findings in ADD patients have revealed abnormally reduced spectral coherence in alpha and beta $(13-20 \mathrm{~Hz})$ rhythms between posterior electrode pairs at the group level (Jelic et al., 2000; Jeong et al., 2004). Alternative techniques sensitive to both linear and nonlinear interrelatedness (e.g., synchronization likelihood, phase lag index, directed transfer function, linear lagged connectivity, etc.) may cross-validate topography of the effects, while source analysis localizes them at intrahemispherical and inter-hemispherical levels (Babiloni et al., 2016b, 2017; Stam et al., 2007; Blinowska et al., 2017). More variable and less reliable are those readouts at delta and theta coherences (Knott et al., 2000), possibly due to the complex topographical pattern of the effects.

For applications in intervention clinical trials, deterioration of EEG rhythms can be longitudinally investigated over time (e.g. about 12-24 months) in groups of MCI and ADD subjects and there is a body of existing evidence permitting predicted outcomes of an efficacious therapeutic on the electrophysiological read outs: (1) increased delta-theta and decreased alpha-beta rhythms at parieto-occipital electrodes (Coben et al., 1985); (2) increased theta and decreased beta rhythms at temporal and temporooccipital electrodes (Jelic et al., 2000); and (3) increased delta and decreased alpha 1 in parieto-occipital sources (Babiloni et al., 2014b). Approved symptomatic treatments, e.g. Acetylcholinesterase inhibitor drugs (i.e. enhancing the cholinergic tone) showed a reduction in deterioration of delta (Balkan et al., 2003; Gianotti et al., 2008), theta (Adler et al., 2004; Gianotti et al., 2008), and alpha frequency rhythms over time (Balkan et al., 2003). If beneficial effects are observed in short-term studies, they may predict longer-term therapeutic efficacy (Adler et al., 2004; Babiloni et al., 2013), although as the effects reported in these studies have been mixed, additional investigation is necessary.

Of note, the mentioned EEG biomarkers have been validated in ADD patients with other established biomarkers including, cortical blood hypoperfusion and synaptic dysfunction and atrophy in the posterior cortex (Fernandez et al., 2003; Babiloni et al., 2015) revealed by PET and structural MRI studies, respectively.

\section{MEG}


The mentioned EEG biomarkers at the group level were cross-validated using highresolution MEG techniques. Compared to Nold subjects, ADD and MCI groups showed an increase of delta/theta rhythms and a decrease of alpha rhythms (Fernández et al., 2006a), more pronounced in the former than the latter. Compared to monodomain (amnesic) MCI, multidomain MCI patients showed more similarities with the ADD group (López et al., 2014). Furthermore, MCI patients with higher delta rhythms had greater risk of progressing to dementia (López et al., 2014). Moreover, both single and multiple-domain MCI groups exhibited a decreased functional connectivity affecting medial temporal and parietal regions within alpha and beta frequency bands (Cuesta et al., 2015). At the brain connectome level, combined MRI and MEG techniques in prodromal AD patients unveiled both structural disconnection and derangement of alpha rhythms in the default mode network (DMN) involving posterior cingulate, precuneus, angular gyrus, and medial prefrontal cortex (Garcés et al., 2014). Furthermore, MCI patients with abnormal CSF p-tau and A $\beta 42$ levels compared with controls (i.e. MCI patients with normal CSF markers) exhibited abnormal functional connectivity among limbic structures such as the anterior/posterior cingulate cortex, orbitofrontal cortex, and medial temporal areas in different frequency bands (Canuet et al., 2015). Moreover, they exhibited a reduction in posterior cingulate functional connectivity mediated by p-tau, associated with impaired axonal integrity of the hippocampal cingulum (Canuet et al., 2015). Finally, cognitively normal older adults positive to amyloid PET diagnostic markers of $\mathrm{AD}$ showed an abnormal functional connectivity of the precuneus (Nakamura et al., 2017).

Another validation of MEG techniques concerned nonlinear and complex features of neural systems generating brain rhythms in AD. Lempel-Ziv complexity and approximate entropy markers unveiled reduced entropy and complexity in MEG rhythms in groups of ADD patients compared with MCI and Nold subjects (Fernández et al., 2010; Azami et al., 2017).

\subsection{Resting state EEG/MEG rhythms in $\mathrm{AD}$ at the individual level}

\section{$E E G$}

Stratification of ADD and MCI individuals is an important step in observational (e.g., prevention studies) and intervention clinical trials. An exciting research line may test this biomarker feature on amplitude or source activity of resting state (eyes-closed) cortical EEG rhythms. A bulk of existing evidence highlights the under-utilised power of EEG information in dementia patient stratification and warrants further investment to fully validate these applications for clinical utility. Global delta and alpha coherences between electrode pairs 
successfully classified ADD compared with DLB people with an accuracy of $75-80 \%$. Combined EEG power density and coherence reached $90 \%$ in the discrimination of ADD vs. Nold and ADD vs. PDD subjects (Engedal et al., 2015). A similar approach reached a classification accuracy of $100 \%$ in small populations of ADD, PDD/DLB, and frontotemporal dementia patients.

A clinical study combining EEG data with neuropsychological, clinical, neuroimaging, cerebrospinal fluid, and visual readouts of EEG data permitted a classification accuracy of $87 \%$ in the discrimination between ADD, PDD, and DLB (Dauwan et al., 2016b). Furthermore, a combination of linear and nonlinear measures of interdependence of rsEEG rhythms reached a classification accuracy of $80-85 \%$ in the discrimination of Nold and MCI individuals (Dauwels et al., 2011). A classification accuracy around $80 \%$ is reached using cortical EEG source estimates in the discrimination between Nold subjects vs. ADD/DLB/PDD patients and ADD vs. PDD patients with an accuracy of 85-90\% (Babiloni et al., 2017). Such accuracy drops under $80 \%$ in the classification of individuals with prodromal stages of those dementing disorders (Babiloni et al., 2017).

Another interesting clinical effort should focus on testing the predictive value of EEG biomarkers at about 6-24 months. Previous studies have showed encouraging results with the following variables: (1) combined alpha-theta power density and mean frequency from left temporal-occipital regions (Jelic et al., 2000); (2) anterior localization of alpha sources (Huang et al., 2000); (3) high temporal delta sources (Rossini et al., 2006); (4) high theta power density; and (5) low posterior alpha power density (Luckhaus et al., 2008).

\section{MEG}

Again, the mentioned EEG biomarkers at the individual level were cross-validated using high-resolution MEG techniques. A seminal MEG study showed that augmented delta activity in posterior parietal and precuneus cortices was negatively correlated with cognitive status and distinguished with classification accuracy $>80 \%, 23$ MCI patients from 35 AD patients with different severity scores and 24 controls (Fernández et al., 2013).

In other exploratory MEG studies carried out in small groups of ADD vs. Nold individuals (e.g. mostly around 20 subjects for group), nonlinear estimates reached accuracies of about 80-87\% (Gómez et al., 2009). Furthermore, Lempel-Ziv complexity estimates combined with age reached accuracies of about $95 \%$ in the discrimination between ADD and MCI individuals with less success between Nold and MCI ones (Fernández et al., 2010). In another trial, entropy estimates reached accuracies of $83.9 \%$ and $65.9 \%$ in the discrimination 
of ADD and MCI subjects from Nold subjects, respectively (Bruña et al., 2012). These preliminary MEG results on at risk populations were challenged in a study that compared 102 MCI patients to 82 age-matched controls and found that mutual information and functional connectivity estimates reached a discriminative accuracy of 79-83\% (Maestú et al., 2015).

Other cross-validation research may relate resting state MEG readouts and AD physiopathological markers. Along this line, posterior MEG delta rhythms correlated with hippocampal atrophy, and a combination of rsMEG and MRI volumetric data allowed a correct classification of $87.1 \%$ (Fernández et al., 2003). In a first follow-up study, the estimated relative risk of conversion from MCI to ADD dementia was increased by $35 \%$ in those MCI patients with augmented posterior parietal delta activity (Fernández et al., 2006c). In a second follow-up study, a combination of left hippocampal volume, occipital cortex theta power, and clock drawing copy subtest scores predicted conversion from MCI to AD dementia with an accuracy of 97\% (López et al., 2016).

\subsection{Event-related EEG/MEG activity in $\mathrm{AD}$ at the group level}

Amplitude or source activity of event-related EEG activity probes the mechanisms of synchronization/desynchronization and coupling/decoupling of thalamocortical and ascending activity systems during sensory and cognitive-motor information processes and can unveil the progressive effects of $\mathrm{AD}$ and intervention at the group level, especially at early disease stages. Previous evidence encourages investment in more clinical research in this area. Panel 3 provides more details on the typical experimental paradigms used in this research.

In an auditory oddball task (i.e. ignore frequent stimuli, react to rare "target" stimuli) involving attention and short-term episodic memory, P50 amplitude following frequent stimuli ( $\mathrm{P}=$ positive; peak at around $+50 \mathrm{~ms}$ poststimulus) was greater in MCI compared with Nold subjects. It was also greater in multidomain/progressing than monodomain/stable MCI subjects (Golob et al., 2007). Furthermore, P300 amplitude following rare stimuli was smaller and its peak latency longer in MCI and ADD patients compared with Nold subjects, even across task difficulty and sensory modalities (Golob et al., 2007; Polich and CoreyBloom, 2005). The P300 has been shown sensitive to AD neuropathology (Morgan and Murphy, 2002), although its latency also increases over time (about 1-2 ms/year) in physiological aging (Iragui et al., 1993). 
ERP components showed differences in Nold and ADD subjects during the analysis of (1) semantic/linguistic stimuli and (2) repeated words. In Nold subjects, parietotemporal $\mathbf{N 4 0 0}$ is larger in amplitude in response to meaningful stimuli that are semantically incongruous, regardless of stimulus type (words vs. images) and types of mismatch (semantic vs. phonological; Kutas and Federmeier, 2011). This effect is linked to semantic memory and differs from the mid-frontal N400 (FN400) related to familiarity-related memory processes (Rugg and Curran, 2007). Physiological aging reduces the modulation of N400 by semantic incongruity, but less than in pathological aging; indeed, both above factors modulating N400 were reduced or abolished in AD patients with mild dementia (Olichney et al., 2006).

In healthy subjects, ERP data also exhibit a significant word repetition effect for both incongruous and congruous words, namely an attenuation of their amplitude. Noteworthy, the attenuation of the N400 for the repetition of incongruous words was poor in MCI compared with Nold subjects (Olichney et al., 2002) and predicted subsequent progression to AD dementia. Such observations largely applied across modalities, make N400 a promising biomarker to assess disease severity and progression (Olichney et al., 2011). In the same line, the attenuation of a widespread posterior P600 for the repetition of congruous words was poor in AD patients with mild dementia compared with Nold subjects (Olichney et al., 2006) and predicted subsequent progression from MCI to AD dementia.

ERP components also point to differences in Nold and AD subjects during episodic memory tasks. In healthy subjects, the P600 is a late positive component (LPC) observed during both memory encoding and retrieval (recall or recognition) of words. The P600 amplitude normally increases for previously presented words ("old" words) successfully retrieved, while it decreases when words are repeated in a predictable, fixed context. Previous studies with ADD and MCI patients revealed that the P600 repetition effect is greatly reduced or absent in ADD and present but delayed in MCI non-converters (Olichney et al., 2002). It can be speculated that early detection or diagnosis of AD may be enhanced by including semantically congruous and incongruous word pairings within a repetition paradigm, allowing assessment of P600 as well as N400.

The above "cognitive" ERP components and others were also able to predict $\boldsymbol{A D}$ progression over time at the group level. Compared with Nold or stable MCI over time, progressing-to-dementia MCI showed increased P50 and N100 amplitude, decreased N200 amplitude (Papaliagkas et al., 2011), increased N200 latency (Missonier, 2007), increased P300 latency (Lai et al., 2010), decreased N400 and P600 (Olichney et al., 2002), and decreased mismatch negativity (MMN) reflecting the automatic detection of changes in the acoustic 
environment (Lindin et al., 2013). Furthermore, pre-symptomatic autopsy-proven older adults (Olichney et al., 2013) and AD mutation carriers (Golob et al., 2009) exhibited reduced N400 to semantic processing (Bobes et al., 2010) and an abnormal topography of P200-P300 (Quiroz et al., 2011) during episodic memory almost 10 years prior to clinical presentation.

The above "cognitive" ERP components monitored AD progression over time at the group level. In longitudinal studies, MCI and ADD patients (untreated with cholinergic therapy) showed changes over time in the MMN, P300, N400, and P600 (Lindin et al., 2013; Papaliagkas et al., 2011).

ERPs were also sensitive to cholinergic therapy (Donepezil or Rivastigmine) in ADD patients, after both short (days/few weeks) and longer periods (3 months-1 year). Specifically, the beneficial effects of the treatment on brain function were mainly observed as a reduction of auditory P300 peak latencies that related to an improvement on cognitive tests (Onofrj et al., 2003).

EROs were repeatedly investigated in oddball tasks. Frontal delta EROs responses (visual and auditory) were attenuated in ADD patients compared with Nold subjects (Yener et al., 2007). Also, theta EROs showed lower frontal phase-locking values in the former than the latter (Güntekin et al., 2008). This effect was less evident or negligible when visual and auditory stimuli were given with no task requested (Yener et al., 2013). EROs were also sensitive to AD progression over time and cholinergic therapy. Progressing-to-dementia MCI patients showed lower theta and beta EROs compared with stable MCI patients (Missonier et al., 2007; Jiang et al., 2015; Hedges et al 2016). In cross-sectional studies, EROs in oddball task demands showed higher frontal theta phase-locking across repeated events (Yener et al., 2007) and greater fronto-parietal alpha coherences in ADD patients treated with AChEIs compared with non-treated ones (Güntekin et al., 2008). With simple visual stimuli with no task, EROs showed improved posterior theta responses in ADD patients treated with AChEIs compared with non-treated ones.

Event-related EEG rhythms were also investigated using a cross-modal episodic memory task. Differences were observed in EEG complexity between untreated ADD and control groups over multiple time scales during task execution (Morison et al., 2013).

Event-related EEG biomarkers have been cross-validated using high-resolution MEG techniques. Compared with Nold subjects, MCI patients showed significantly higher occipital, temporal, and frontal ventral ERMFs during a short-term memory task (Maestú et al., 2008). Furthermore, MCI patients showing significantly higher memory task-related alpha and beta rhythms in a ventral cortical pathway, converted more rapidly to AD dementia (Bajo et al., 
2012a). When such augmented MEG activity was explored using a functional connectivity approach, MCI patients exhibited an increased marker of prefrontal and long-range functional connectivity from alpha to gamma bands (Bajo et al., 2010). Finally, another MEG study pointed to a reduction in the complexity of functional networks involved in memory-related changes (Sternberg task) of widespread theta and alpha rhythms (Ahmadlou et al., 2014).

\subsection{Event-related EEG/MEG biomarkers of $\mathrm{AD}$ at the individual level}

Stratification of ADD and MCI individuals in observational and intervention clinical trials may be based on event-related EEG/MEG biomarkers. A recent study showed that the $M M N$ amplitude successfully discriminated aMCI and Nold individuals with a classification accuracy of 75\% (Lindin et al., 2013).

In the prodromal stage of $\mathrm{AD}$, a reduced word repetition effect on the $\mathbf{P 6 0 0}$ discriminated MCI subjects who converted to dementia compared with "stable" MCI subjects with a classification accuracy of $85 \%$ (Olichney et al., 2002). In another study, oddball P300 latency allowed prediction to $\mathrm{AD}$ dementia with an accuracy of $65 \%$ in subjects with subjective memory complaints (Gironell et al., 2005). Analogously, auditory N200 resulted in a classification accuracy of $91 \%$ in the prediction of MCI patients that converted to ADD within the follow-up at 5 years (Papaliagkas et al., 2008). Furthermore, ERPs (including the P300) accompanying a number-letter paradigm, which employed a visual task with memory and other cognitive demands, predicted the progression to AD dementia with an accuracy of 70$78 \%$ in MCI subjects (Chapman et al., 2011).

Oddball parietal delta EROs reached a classification accuracy of $76 \%$ in the discrimination of ADD patients vs. Nold subjects (Polikar et al., 2007).

Other ERP components exhibited an interesting prediction value. In MCI subjects, P200 and N200 ERP latencies as well as EROs recorded during attention and memory demands predicted cognitive decline after 1 year with an accuracy of $93 \%, 78 \%$, and $72 \%$, respectively (Missonnier et al., 2007). The combination of the three EEG markers correctly predicted the outcome of $90 \%$ of MCI cases.

An approach based on permutation entropy in MEG reached an accuracy of $98.4 \%$ in the classification of 18 MCI vs. 19 Nold controls using MEG activity related to working memory as an input to a probabilistic network classifier (Amezquita-Sanchez et al., 2016).

\subsection{Sleep EEG biomarkers of AD}


It is well known that sleep plays a crucial role in the processes of learning and memory, so sleep EEG biomarkers are of great interest in AD research. Converging evidence shows that non-rapid eye movement (NREM) stages of sleep are affected by AD processes. In ADD patients, EEG rhythms are characterized by excessive sleep fragmentation (Yulug et al., 2017), associated with a decrease in slow-waves in NREM sleep stages. In those NREM stages, ADD patients showed a significant (>40\%) reduction in K-complex density over the frontal areas, correlated with their cognitive involvement (De et al., 2017). Furthermore, total frontal EEG slow-wave activity predicted reduced cerebrospinal (CSF) A $\beta 1-42$ levels in preclinical AD subjects, when controlling for age and ApoE status (Varga et al., 2016). Moreover, sleep spindles were reported to decrease in ADD patients. More recently, a significant decrease in parietal fast spindle density was also reported in ADD and MCI patients, and this correlated with cognitive performance (Gorgoni et al., 2016). Finally, MCI and ADD patients showed poor Cyclic Alternating Pattern in non-REM sleep (CAP; Parrino et al., 2012; Ferri et al., 2008), as a general difficulty to produce synchronized EEG slow waves underlying neuroplasticity during sleep (Maestri et al., 2015).

\section{BACK-TRANSLATION TO PRECLINICAL ELECTROPHYSIOLOGY}

Out of the 244 compounds (413 clinical trials) developed for AD between 2002 and 2012, only one (0.4\%) was approved (Cummings et al., 2014). Therefore, there is a dramatic need for high-construct validity in vivo efficacy assays and functional in vivo biomarkers in rodent models of $\mathrm{AD}$ as well as in vitro systems that fill the void of back-translational validity (Walsh et al., 2017). For this purpose, electrophysiology has ideal features with highly stratified spatial (from micro to macroscale) and millisecond temporal resolution (Booth et al., 2016; Drinkenburg, 2015; Jyoti et al., 2015; Scott et al., 2016). Electrophysiological readouts can be more readily interpreted in terms of functional relevance of the (compromised) brain in its full complexity (Palop and Mucke, 2016; Walsh et al., 2017).

\subsection{Electrophysiology in neuronal cultures}

Neurons isolated from rodent brains and cultured in vitro give insights into how disease pathology impacts the intrinsic functional output of a neuron or a simple network and can provide meaningful insights into more complex hierarchical systems. Cellular changes in activity can often be extrapolated up to those seen in vivo or in patients and can be recorded 
using single electrodes to look at single cell activity or multielectrode arrays (MEAs) to observe networks. Neurons have an intrinsic necessity to network to survive in vivo and isolated neurons in culture will connect to each other and become synaptically networked with synchronised activity in a matter of days in vitro. Neurotoxic species such as synthetic peptides (commonly oligomeric $\mathrm{A} \beta$ ), cell-derived, and human brain-derived material can be successfully used in probing the effect of AD-relevant toxic species on electrophysiological activity of neuronal cultures at spatial microscales using single or multielectrode recordings. Synthetic aggregated $A \beta$ oligomers robustly alter neuronal excitability and survival and can be recorded with patch clamp techniques (Ferreira et al., 2011) but human AD brain-derived A $\beta$ is more potent and has electrophysiological consequences at more physiologically relevant concentrations, but is more methodologically challenging due to the isolation of specific $\mathrm{A} \beta$ fractions (Shankar et al., 2008).

It is also possible to generate primary neuronal cultures from transgenic animals overexpressing $A \beta$ or Tau whose neurons also display increased intrinsic neural excitability associated with AD-relevant pathological challenges (e.g. Sanchez et al., 2012). Synaptic changes are observed in culture and in-vivo but there is no proteinopathy in $2 \mathrm{D}$ culture.Tau seeding models can be used to mimic the neurodegenerative AD process more closely (Peeraer et al., 2015). Newer, three dimensional cultures where neurons are embedded in a 3D matrix or cultured in spheroids do show more realistic pathological aggregations including both intracellular tau tangles and extracellular amyloid plaques (reviewed in D'Avanzo et al, 2015), however the functional analysis of these cultures using electrophysiology remains to be reported and may be more complex due to difficulty in accessing cells through the matrix. Most contemporary AD genetic models are mice carrying a human amyloid precursor protein (hAPP) mutation (usually APPswe) alone or in combination with presenilin-1 (PS-1) mutation and/or neurofibrillary tangles generated by expression of human MAPT (Onos et al., 2016). APP/(PS1) mice show human AD-like amyloid plaques in the cortex and hippocampus, and age-related memory impairment, but no neuronal loss unless several mutations are combined as in a so-called 5xFAD mouse model (Oakley et al., 2006). They may also show synaptic loss correlative with neurodegenerative processes (Mucke and Selkoe, 2012). More recently developed knock-in transgenic mouse models are considered a closer model of AD pathology since they do not over-express APP (Zhang et al., 2016). However, no transgenic AD mouse model reproduces all main pathophysiological (i.e., $\mathrm{A} \beta$ or tau) and progressive cognitive and behavioral deficits like AD patients (reviewed in Gotz et al., 2008). Furthermore, these strains 
can be considered as models of pre-symptomatic to mild AD. Beyond them, new transgenic rat models or intraventricular/intracerebral $A \beta$ infusion in wild type rodents can be considered for AD electrophysiological research (e.g., Do Carmo and Cuello, 2013). Until very recently, studies of human neurons have been limited to immortal cell lines (e.g. SY-5Y neuroblastoma lines). They can be differentiated into more neuron-like cells and are very useful for studying the integrity of cholinergic activity, as well as testing compounds on cholinergic currents; however, they lack some intrinsic functional capabilities of primary neurons (Kar et al., 2004). Recent advances in induced pluripotent stem cell (iPSC) technology make it possible to perform living human neuronal studies in a dish (Takahashi et al., 2007). Differentiation protocols can generate over 50 different types of neurons and glia cells relevant for human disease modeling and hypothesis testing (Taber and Studer, 2014) as a function of genotyping and phenotypes (Mertens, et al 2015). Human neurons can be studied using similar approaches to the rodent neuronal study methods outlined.

These cell culture methodologies provide practical screening opportunities for pharmacological agents that can modify functional deficits driven by pathology in vitro.

\subsection{Electrophysiology in brain slices}

Promising preclinical AD research can be performed by recording electrophysiological signals from acute brain slices with locally intact neuronal networks formed by endogenous cells and functional connections in specific brain regions in isolation without 'noise' due to other complex connections. This approach allows mechanistic probing studies of pharmacological agents and also cellular communication between neurons and the brain's other resident immune cells, including glia and microglia. The effects of A $\beta 42$ species on microglia (but not infiltrating monocytes) have been studied in this system (Villegas-Llerena et al., 2016).

Acute brain slices (e.g., from hippocampus and cortex) can be derived from: (1) wild type rodents challenged with acute application of $A \beta 42$, tau, modulatory neurotransmitters (e.g. Acetylcholine, glutamate) or therapeutic agents by intraventricular/intracerebral injections (e.g. Xu et al 2016) or (2) transgenic AD mouse models at different ages and stages of the pathology. Analysis may focus on effects of those agents on resting membrane potential, firing frequency and after-hyperpolarization magnitude, neural hyperexcitability, synaptic plasticity including long-term potentiation (LTP) and depression (LTD), and many cognitively relevant network oscillations at beta $(20-30 \mathrm{~Hz})$ and gamma $(30-80 \mathrm{~Hz})$ frequencies (e.g. Hazra et al., 2013; Tamagnini et al., 2015). Expected results should reveal progressive impairment in neural 
synchronization and functional connectivity in hippocampal and cortical networks with reduced LTP and beta $(20-30 \mathrm{~Hz})$ and gamma $(30-80 \mathrm{~Hz})$ frequency oscillations (Driver et al 2007). However, some studies suggest caution as $A \beta$ monomer/oligomer distribution in a given slice can reveal strongly variable deficits in LTP (Rammes et al., 2011). Furthermore, in-vivo mouse AD models showed mixed results concerning the reduction of those beta and gamma rhythms, possibly in relation to brain regions of interest, age, strain, and disease progression (Gurevisius et al., 2013, Papazoglou et al., 2016).

Another promising preclinical assay for AD research utilises the Utah array to record electrophysiological signals to map changes in synchronization in longer-range interactions e.g. CA3 to CA1 in the hippocampus, or between deep and superficial layers in the cortex in slices (e.g. Ainsworth et al., 2016). Current source density analysis can reveal changes in brain neural synchronization while Granger analysis can determine interregional directional functional coupling/decoupling within recording contacts in a brain slice (Parsons et al., 2007).

\subsection{Electrophysiology in freely behaving rodents}

In behaving rodent $\mathrm{AD}$ models, abnormal electrophysiological brain activity (including ERPs and sleep) can be successfully recorded at the spatial mesoscale and macroscale by single neuron activity and LFP/EEG, respectively (Drinkenburg 2015; Scott et al., 2016). This approach has an obvious special "back-translational" value with reference to AD clinical symptoms (Palop and Mucke, 2016; Walsh et al., 2017). Even with the mentioned limitations of transgenic rodents (Drummond and Wisniewski, 2017), these animal models allow investigation of the direct relationship between $\mathrm{AD}$ neuropathology (progression) and its neurophysiological effects on brain neural networks (Drinkenburg et al., 2016; Palop and Mucke, 2016). Furthermore, although few studies have been published that use EP/ERP paradigms in AD rodent models (Gurevicius et al., 2013; Takahashi, 2013; Laursen et al., 2014; Drinkenburg et al., 2016; Zhang et al., 2016; Walsh et al., 2017), preclinical validation of the such paradigms is well established and they can be reliably used (Drinkenburg et al., 2016; Leiser et al., 2011).

Keeping in mind this premise, a primary electrophysiological research line may clarify effects of $\mathrm{A} \beta$ on brain rhythms in $\mathrm{AD}$ rodent models. Indeed, hippocampal theta phase-gamma amplitude coupling was reduced in A $\beta$-overproducing 5XFAD transgenic mice and TgF344AD transgenic rats (Stoiljkovic et al., 2017). Furthermore, APP23 $\times$ PS45 mice showed a severely disrupted coherence of calcium transients and LFPs as an impairment of cortical 
functional connectivity (Busche et al., 2015). Moreover, coherence and cross-frequency coupling at theta-gamma rhythms characterized AD processes in rodents (Ahnaou et al., 2014; Fries 2015; Palop and Mucke, 2016; Walsh et al., 2017) and the effects of a beneficial intervention in A $\beta$-overproducing 5XFAD mice (Iaccarino et al., 2016).

Another important electrophysiological research line may characterize firing and oscillatory activities of hippocampal pyramidal neurons in transgenic AD mouse models in relation to learning and plasticity (Cayzac et al., 2015). In this line, normal hippocampal LTP induction but accelerated decay was found in freely moving aged APP/PS1 mice with robust brain amyloidosis (Gureviciene et al., 2004). Furthermore, human Aß selectively disrupted hippocampal LTP induction before amyloid plaque formation in McGill-R-Thy1-APP transgenic rats (Qi and co-workers, 2014). It also altered oscillatory responses to highfrequency stimulation (Kalweit et al., 2015). In the same line, no changes in hippocampal theta oscillations were found in freely moving APP/PS1 mice before significant amyloid plaque formation (Gurevicius et al., 2013).

Another electrophysiological research line may enhance our understanding of molecular and cellular mechanisms of AD-related enhanced excitability in rodents (Minkeviciene et al., 2009; Gurevicius et al., 2013; Palop and Mucke, 2016; Siwek et al., 2015) and the relative optimal anti-epileptic treatment (Ziyatdinova et al., 2011; Rong, 2016). Indeed, APP (Palop et al., 2007), APP/PS1 (Minkeviciene et al., 2009) and tau (Garcia-Cabrero et al., 2013) transgenic mice display frequent epileptic seizures (Born et al., 2015; Subota et al., 2017). In addition, focal epileptic or epileptiform activity in those mice may account not only for cognitive deficits (Palop and Mucke, 2010) but might also relate to early amyloid accumulation (Bero et al., 2011) and trans-synaptic spread of tau pathology (Yamada et al., 2014).

This preclinical research line has remarkable translational value. A bulk of evidence has shown an increased risk of overt epileptic seizures or subclinical, non-convulsive, epileptiform-like EEG signatures in AD patients with fast clinical deterioration over time when compared to controls (Horváth et al., 2016; Vossel et al., 2016). Furthermore, convulsive seizures are 10 times more frequent in $\mathrm{AD}$ patients than the general population (Horváth et al., 2016), while epilepsy is 87 times more frequent in AD patients with early than the late-onset disease (Scarmeas et al., 2009). Moreover, cognitive disorders appear 5.5 years earlier in AD patients with epileptiform discharges than AD controls (Vossel et al., 2013). Most of those discharges were observed during sleep, especially the non-rapid eye movement one (Horváth et al., 2017). 
Panel 4 illustrates the most promising conditions of LFP/EEG recordings in freely behaving rodents.

\section{CONCLUDING RECOMMENDATIONS}

Electrophysiology is a multi-scale methodology suited to probing the effects of AD neuropathological processes (e.g. A $\beta 1-42$, phospho tau, neurodegeneration of cholinergic systems, dysregulation of glutamate homeostasis) and disease-modifying drugs on synchronization/desynchronization and coupling/decoupling of brain neural activity in preclinical and clinical research.

For preclinical AD research, we recommend the following methodologies:

(1) At the microscale, in-vitro studies of membrane ionic currents and potentials at a single neuronal cell or slices of hippocampal tissue (Ferreira et al., 2011).

(2) At the mesoscale, in-vivo LFP studies of synaptic dysfunction and neural overexcitation in transgenic rodent models complementing and cross-validating cell and slice electrophysiology studies (Drinkenburg et al., 2016; Palop and Mucke 2016; Walsh et al., 2017).

(3) At the macroscale, in-vivo scalp and intracranial EEG/ERP studies of aberrant brain neural synchronization (e.g., increased risk of epilepsy) and functional thalamocortical and corticocortical connectivity as revealed by linear and nonlinear measures (Vossel et al., 2016; Horváth et al., 2017; Lam et al., 2017).

The use of EEG in clinical AD research is impressive (see Table 1 in Supplementary material). Particularly abundant is the EEG/MEG research in patients with mild ADD during quiet wakefulness, which can be virtually developed in any hospital EEG lab in a non-invasive and cost-effective manner. Novel, smart technologies emerging may also permit monitoring outside of the clinical setting soon. Relative topographical spectral (frequency domain) biomarkers may monitor the progression of the disease over years and therapy response. Indeed, this experimental condition is repeatable, noninvasive, and comparable during different stages of the disease and cognitive status.

We also recommend EEG/MEG recordings and analysis (frequency and time domains) of data obtained in event-related paradigms in patients with preclinical and prodromal AD stages. Candidate biomarkers may be the reduction in frontal delta/theta and posterior alpha rhythms 
and the amplitude decrease of the late positive potentials (e.g., oddball paradigm). Other promising candidate biomarkers probe reduction in functional cortical connectivity and complexity in several frequency bands at prodromal and dementia stages of the disease. Those candidate biomarkers might be used as "gatekeepers" for vast populations of seniors at risk of AD. Necessary conditions for such use are their good sensitivity (> 80\%) to prodromal or mild dementia stages of $\mathrm{AD}$ and consensus in international initiatives to overcome the current heterogeneity of methodological approaches for data recording and analysis. In addition, specificity as well as correlation with other biomarkers will thus have to be determined. Exemplary references for these future studies are (1) diagnostic guidelines of the IWG-2 for AD (Dubois et al., 2014), in particular the recommendations for diagnostic and topographical biomarkers and (2) international projects such as IMI PharmaCog (www.pharmacog.org), ADNI (http://www.adni.loni.usc.edu), and ENIGMA (http://www.enigma.ini.usc.edu).

\section{REFERENCES}

Adler, G., Brassen, S., Chwalek, K., Dieter, B., Teufel, M., 2004. Prediction of treatment response to rivastigmine in Alzheimer's dementia. J Neurol Neurosurg Psychiatry. 75, 292-4.

Ahmadlou, M., Adeli, A., Bajo, R., Adeli, H., 2014. Complexity of functional connectivity networks in mild cognitive impairment subjects during a working memory task. Clin Neurophysiol. 125, 694-702.

Ahnaou, A., Huysmans, H., Jacobs, T., Drinkenburg, W.H.I.M., 2014. Cortical EEG oscillations and network connectivity as efficacy indices for assessing drugs with cognition enhancing potential. Neuropharmacology. 86, $362-77$.

Ainsworth, M., Lee, S., Kaiser, M., Simonotto, J., Kopell, N.J., and Whittington, M.A., 2016. GABAB receptormediated, layer-specific synaptic plasticity reorganizes gamma-frequency neocortical response to stimulation. Proc Natl Acad Sci U S A. 113, E2721-9.

Amezquita-Sanchez, JP., Adeli, A., Adeli, H., 2016. A new methodology for automated diagnosis of mild cognitive impairment (MCI) using magnetoencephalography (MEG). Behav Brain Res. 305, 174-80.

Azami, H., Fernández, A., Escudero, J., 2017. Refined multiscale fuzzy entropy based on standard deviation for biomedical signal analysis. Med Biol Eng Comput. 55, 2037-2052.

Babiloni, C., Cassetta, E., Dal Forno, G., Del Percio, C., Ferreri, F., Ferri, R., Lanuzza, B., Miniussi, C., Moretti DV., Nobili, F., Pascual-Marqui, RD., Rodriguez, G., Romani, GL., Salinari, S., Zanetti, O., Rossini, PM., 2006a. Donepezil effects on sources of cortical rhythms in mild Alzheimer's disease: Responders vs. Non-Responders. Neuroimage. 31, 1650-65.

Babiloni, C., Ferri, R., Binetti, G., Cassarino, A., Dal Forno, G., Ercolani, M., Ferreri, F., Frisoni, G.B., Lanuzza, B., Miniussi, C., Nobili, F., Rodriguez, G., Rundo, F., Stam, C.J., Musha, T., Vecchio, F., Rossini, P.M., 2006 b. Fronto-parietal coupling of brain rhythms in mild cognitive impairment: A multicentric EEG study. Brain Res Bull. 69, 63-73.

Babiloni, C., Benussi, L., Binetti, G., Bosco, P., Busonero, G., Cesaretti, S., Dal Forno, G., Del Percio, C., Ferri, R, Frisoni, G., Ghidoni, R., Rodriguez, G., Squitti, R., Rossini, P.M., 2006c. Genotype (cystatin C) and EEG phenotype in Alzheimer disease and mild cognitive impairment: a multicentric study. Neuroimage. 29, 948-64.

Babiloni, C., Carducci, F., Lizio, R., Vecchio, F., Baglieri, A., Bernardini, S., Cavedo, E., Bozzao, A., Buttinelli, C., Esposito, F., Giubilei, F., Guizzaro, A., Marino, S., Montella, P., Quattrocchi, C.C., Redolfi, A., Soricelli, A., Tedeschi, G., Ferri, R., Rossi-Fedele, G., Ursini, F., Scrascia, F., Vernieri, F., Pedersen, T.J., Hardemark, H.G., Rossini, P.M., Frisoni, G.B., 2013. Resting state cortical electroencephalographic rhythms are related to gray matter volume in subjects with mild cognitive impairment and Alzheimer's disease. Hum Brain Mapp. 34, 142746. 
Babiloni, C., Del Percio, C., Lizio, R., Marzano, N., Infarinato, F., Soricelli, A., Salvatore, E., Ferri, R., Bonforte, C, Tedeschi, G., Montella, P., Baglieri, A., Rodriguez, G., Famà, F., Nobili, F., Vernieri, F., Ursini, F., Mundi, C., Frisoni, G.B., Rossini, P.M., 2014. Cortical sources of resting state electroencephalographic alpha rhythms deteriorate across time in subjects with amnesic mild cognitive impairment. Neurobiol Aging. 35, 130-42.

Babiloni, C., Del Percio, C., Boccardi, M., Lizio, R., Lopez, S., Carducci, F., Marzano, N., Soricelli, A., Ferri, R., Triggiani, A.I., Prestia, A., Salinari, S., Rasser, P.E., Basar, E., Famà, F., Nobili, F., Yener, G., Emek-Savaş, DD., Gesualdo, L., Mundi, C., Thompson, P.M., Rossini, P.M., Frisoni, G.B., 2015. Occipital sources of resting-state alpha rhythms are related to local gray matter density in subjects with amnesic mild cognitive impairment and Alzheimer's disease. NeurobiolAging. Feb;36(2), 556-70.

Babiloni, C., Del Percio, C., Caroli, A., Salvatore, E., Nicolai, E., Marzano, N., Lizio, R., Cavedo, E., Landau, S., Chen, K., Jagust, W., Reiman, E., Tedeschi, G., Montella, P., De Stefano, M., Gesualdo, L., Frisoni, G.B., Soricelli, A., 2016. Cortical sources of resting state EEG rhythms are related to brain hypometabolism in subjects with Alzheimer's disease: an EEG-PET study. NeurobiolAging. 48, 122-134.

Babiloni, C., Del Percio, C., Lizio, R., Noce, G., Cordone, S., Lopez, S., Soricelli, A., Ferri, R., Pascarelli, M.T., Nobili, F., Arnaldi, D., Aarsland, D., Orzi, F., Buttinelli, C., Giubilei, F., Onofrj, M., Stocchi, F., Stirpe, P., Fuhr, P., Gschwandtner, U., Ransmayr, G., Caravias, G., Garn, H., Sorpresi, F., Pievani, M., Frisoni, G.B., D'Antonio, F., De Lena, C., Güntekin, B., Hanoğlu, L., Başar, E., Yener, G., Emek-Savaş, D.D., Triggiani, A.I., Franciotti, R., De Pandis, M.F., Bonanni, L. 2017. Abnormalities of cortical neural synchronization mechanisms in patients with dementia due to Alzheimer's and Lewy body diseases: an EEG study. Neurobiol Aging. 55, 143-158.

Bajo, R., Maestú, F., Nevado, A., Sancho, M., Campo, P., Gutiérrez, R., Gil, P., Pereda, E., Van Dijk, B., Pozo, F.. 2010. Functional connectivity in mild cognitive impairment during a memory task: implications for the disconnection hypothesis. J Alzheimers Dis. 22, 183-93.

Bajo, R., Castellanos, N.P., Cuesta, P., Aurtenetxe, S., Garcia-Prieto, J., Gil-Gregorio, P., Del-Pozo, F., Maestu, F., 2012a. Differential patterns of connectivity in progressive mild cognitive impairment. Brain Connect. 2, 21-4.

Balkan, S., Yaras, N., Mihci, E., Dora, B., Agar, A., \& Yargicoglu, P., 2003. Effect of donepezil on EEG spectral analysis in Alzheimer's disease. Acta Neurol Belg, 103, 164-169.

Bero, A.W., Yan, P., Roh, J.H., Cirrito, J.R., Stewart, F.R., Raichle, M.E., Lee, J.M., Holtzman, D.M., 2011. Neuronal activity regulates the regional vulnerability to amyloid- $\beta$ deposition. Nat Neurosci. 14, 750-6.

Blinowska, K.J., Rakowski, F., Kaminski, M., De Vico Fallani F., Del Percio, C., Lizio, R., Babiloni, C., 2017. Functional and effective brain connectivity for discrimination between Alzheimer's patients and healthy individuals: A study on resting state EEG rhythms. Clin Neurophysiol. 128, 667-680.

Bruña, R., Poza, J., Gómez, C., García, M., Fernández, A., Hornero, R., 2012. Analysis of spontaneous MEG activity in mild cognitive impairment and Alzheimer's disease using spectral entropies and statistical complexity measures. J Neural Eng. 9, 036007.

Bullmore, E., Sporns, O., 2009. Complex brain networks: graph theoretical analysis of structural and functional systems. Nat Rev Neurosci. 10, 186-98.

Busche, M.A., Kekuš, M., Adelsberger, H., Noda, T., Förstl, H., Nelken, I., Konnerth, A., 2015. Rescue of longrange circuit dysfunction in Alzheimer's disease models. Nat Neurosci. 18, 1623-30.

Canuet, L., Pusil. S., López, M.E., Bajo, R., Pineda-Pardo, J., Cuesta, P., Galvez, G., Gaztelu, J.M., Lourido, D., García-Ribas, G., Maestú, F., 2015. Network Disruption and Cerebrospinal Fluid Amyloid-Beta and PhosphoTau Levels in Mild Cognitive Impairment. J Neurosci. 35, 10325-30.

Cayzac, S., Mons, N., Ginguay, A., Allinquant, B., Jeantet, Y., Cho, Y.H., 2015. Altered hippocampal information coding and network synchrony in APP-PS1 mice. Neurobiology of Aging. 36, 3200-13.

Chapman, R.M., McCrary, J.W., Gardner, M.N., Sandoval, T.C., Guillily, M.D., Reilly, L.A., DeGrush, E., 2011. Brain ERP components predict which individuals progress to Alzheimer's disease and which do not. Neurobiol Aging. 32, 1742-55.

Cuesta, P., Garcés, P., Castellanos, N.P., López, M.E., Aurtenetxe, S., Bajo, R., Pineda, J., Bruña, R., Marín, A.G., Delgado, M., Barabash, A., Ancín, I., Cabranes, J.A., Fernandez, A., Del Pozo, F., Sancho, M., Marcos, A., Nakamura, A., Maestú, F., 2015. Influence of the APOE $\varepsilon 4$ Allele and Mild Cognitive Impairment Diagnosis in the Disruption of the MEG Resting State Functional Connectivity in Sources Space. J Alzheimers Dis. 44, 493505 . 
Cummings, J.L., Zhong, K., 2014. Repackaging FDA-approved drugs for degenerative diseases: promises and challenges. Expert Rev Clin Pharmacol. 7, 161-5.

D'Avanzo, C., Aronson, J., Kim, Y.H., Choi, S.H., Tanzi, R.E., Kim, D.Y., 2015. Alzheimer's in 3D culture: challenges and perspectives. Bioessays. 37, 1139-48

Dauwan, M., van der Zande, J.J., van Dellen, E., Sommer, I.E., Scheltens, P., Lemstra, A.W., Stam, C.J., 2016. Random forest to differentiate dementia with Lewy bodies from Alzheimer's disease. Alzheimers Dement (Amst). 19, 99-106.

Dauwels, J., Srinivasan, K., Ramasubba, R.M., Musha, T., Vialatte, F.B., Latchoumane, C., Jeong, J., Cichocki, A., 2011. Slowing and Loss of Complexity in Alzheimer's EEG: Two Sides of the Same Coin? Int J Alzheimers Dis. 2011, 539621.

De, G.L., Gorgoni, M., Reda, F., Lauri, G., Truglia, I., Cordone, S., Scarpelli, S., Mangiaruga, A., D'Atri, A., Lacidogna, G., Ferrara, M., Marra, C., Rossini, P.M., 2017. The Fall of Sleep K-Complex in Alzheimer Disease. Sci Rep. 7, 39688.

Do Carmo, S., Cuello, A. C., 2013. Modeling Alzheimer's disease in transgenic rats. Mol Neurodegener. 8, 37.

Drinkenburg, W.H.I.M., Ruigt, G.S.F., Ahnaou, A., 2015. Pharmaco-EEG studies in animals: an overview of contemporary translational applications. Neuropsychobiology, 72, 151-16.

Drinkenburg, W.H.I.M., 2015. Electroencephalography and Pharmaco-electroencephalography. In: Stolerman IP \& Price LH (eds) Encyclopedia of Psychopharmacology (Second Edition). Springer, Berlin.

Driver, J.E., Racca, C., Cunningham, M.O., Towers, S.K., Davies, C.H., Whittington, M.A., LeBeau, F.E., 2007. Impairment of hippocampal gamma-frequency oscillations in vitro in mice overexpressing human amyloid precursor protein (APP). Eur J Neurosci. 26, 1280-8.

Drummond, E., Wisniewski, T., 2017. Alzheimer's disease: experimental models and reality. Acta Neuropathol. $133,155-175$

Dubois, B., Feldman, H.H., Jacova, C., Hampel, H., Molinuevo, J.L., Blennow, K., DeKosky, S.T., Gauthier, S., Selkoe, D., Bateman, R., Cappa, S., Crutch, S., Engelborghs, S., Frisoni, G.B., Fox, N.C., Galasko, D., Habert, M.O., Jicha, G.A., Nordberg, A., Pasquier, F., Rabinovici, G., Robert, P., Rowe, C., Salloway, S., Sarazin, M., Epelbaum, S., de Souza, L.C., Vellas, B., Visser, P.J., Schneider. L., Stern, Y., Scheltens, P., Cummings, J.L., 2014. Advancing research diagnostic criteria for Alzheimer's disease: the IWG-2 criteria. Lancet Neurol. 13, 61429.

Engedal, K., Snaedal, J., Hoegh, P., Jelic, V., Bo Anderson, B, Naik, M., Wahlund, L.O., Oeksendgaard, A.R., 2015. Quantitative EEG applying the statistical recognition pattern method: A useful tool in dementia diagnostic workup. Dement Geriatr Cogn Disord. 40, 1-12.

Fernandez, A., Arrazola, J., Maestu, F., Amo, C., Gil-Gregorio, P., Wienbruch, C., Ortiz, T., 2003. Correlations of hippocampal atrophy and focal low-frequency magnetic activity in Alzheimer disease: volumetric MR imagingmagnetoencephalographic study. AJNR Am J Neuroradiol. 24, 481-7.

Fernandez, A., Hornero, R., Mayo, A., Poza, J., Gil-Gregorio, P., Ortiz, T., 2006a. MEG spectral profile in Alzheimer's disease and mild cognitive impairment. Clin Neurophysiol. 117, 306-14.

Fernández, A., Turrero, A., Zuluaga, P., Gil, P., Maestú, F., Campo, P., Ortiz, T., 2006b. Magnetoencephalographic parietal delta dipole density in mild cognitive impairment: preliminary results of a method to estimate the risk of developing Alzheimer disease. Arch Neurol. 63, 427-30.

Fernández A, Hornero R, Gómez C, Turrero A, Gil-Gregorio P, Matías-Santos J, Ortiz T., 2010. Complexity analysis of spontaneous brain activity in Alzheimer disease and mild cognitive impairment: an MEG study. Alzheimer Dis Assoc Disord. 24, 182-9.

Fernandez, A., Turrero, A., Zuluaga, P., Gil-Gregorio, P., del Pozo, F., Maestu, F., Moratti, S., 2013. MEG delta mapping along the healthy aging-Alzheimer's disease continuum: diagnostic implications. J Alzheimers Dis. 35, 495-507.

Ferreira, A., Sinjoanu, R.C., Nicholson, A., Kleinschmidt, S., 2011. A $\beta$ toxicity in primary cultured neurons. Methods Mol Biol. 670, 141-53

Ferri, R., Huber, R., Aricò, D., Drago, V., Rundo, F., Ghilardi, M.F., Massimini, M., Tononi, G., 2008. The slowwave components of the Cyclic Alternating Pattern (CAP) have a role in sleep-related learning processes. Neurosci Lett 2008. 432, 228-231. 
Fields, R.D., 2008. White matter in learning, cognition and psychiatric disorders. Trends Neurosci. 31,361-70.

Fries, P., 2015. Rhythms for Cognition, communication through coherence. Neuron. 88, 220-235.

Garcés, P., Angel Pineda-Pardo, J., Canuet, L., Aurtenetxe, S., López, M.E., Marcos, A., Yus, M., Llanero-Luque, M., Del-Pozo, F., Sancho, M., Maestú F., 2014. The Default Mode Network is functionally and structurally disrupted in amnestic mild cognitive impairment - A bimodal MEG-DTI study. Neuroimage Clin. 6, $214-21$.

García-Cabrero, A.M., Guerrero-López, R., Giráldez, B.G., Llorens-Martín, M., Avila, J., Serratosa, J.M., Sánchez, M.P., 2013. Hyperexcitability and epileptic seizures in a model of frontotemporal dementia. Neurobiol Dis. 58, 200-8.

Gianotti, L.R., Künig, G., Faber, P.L., Lehmann, D., Pascual-Marqui, R.D., Kochi, K., Schreiter-Gasser, U., 2008. Rivastigmine effects on EEG spectra and three-dimensional LORETA functional imaging in Alzheimer's disease. Psychopharmacology (Berl). 198, 323-32.

Gironell, A., García-Sánchez, C., Estévez-González, A., Boltes, A., Kulisevsky, J., 2005. Usefulness of p300 in subjective memory complaints, a prospective study. J Clin Neurophysiol. 22, 279-84.

Golob, E.J., Ringman, J.M., Irimajiri, R., Bright, S., Schaffer, B., Medina, L.D., Starr, A., 2009. Cortical eventrelated potentials in preclinical familial Alzheimer disease. Neurology. 73, 1649-55.

Gómez, C., Mediavilla, A., Hornero, R., Abasolo, D., Fernandez, A., 2009. Use of the Higuchi's fractal dimension for the analysis of MEG recordings from Alzheimer's disease patients. Med Eng Phys. 31, 306-13.

Gorgoni, M., Lauri, G., Truglia, I., Cordone, S., Sarasso, S., Scarpelli, S., Mangiaruga, A., D'Atri, A., Tempesta, D., Ferrara, M., Marra, C., Rossini, P.M., De, G.L., 2016. Parietal Fast Sleep Spindle Density Decrease in Alzheimer's Disease and Amnesic Mild Cognitive Impairment. Neural Plast. 2016, 8376108.

Götz, J, Ittner, LM., 2008. Animal models of Alzheimer's disease and frontotemporal dementia. Nat Rev Neurosci. 9, 532-544.

Guntekin, B., Saatci, E., Yener, G., 2008. Decrease of evoked delta, theta and alpha coherences in Alzheimer patients during a visual oddball paradigm. Brain Res, 1235, 109-116.

Gureviciene, I., Ikonen, S., Gurevicius, K., Sarkaki, A., van Groen, T., Pussinen, R., Ylinen, A., Tanila H., 2004. Normal induction but accelerated decay of LTP in APP + PS1 transgenic mice. Neurobiol Dis. 15, 188-95.

Gurevicius, K., Lipponen, A., Tanila, H., 2013. Increased cortical and thalamic excitability in freely moving APPswe/PS1dE9 mice modeling epileptic activity associated with Alzheimer's disease. Cereb Cortex. 23, 114858.

Hazra, A., Gu, F., Aulakh, A., Berridge, C., Eriksen, J.L., Ziburkus, J., 2013.Inhibitory neuron and hippocampal circuit dysfunction in an aged mouse model of Alzheimer's disease. PLoS One. 8, e64318.

Hedges, D., Janis, R., Mickelson, S., Keith, C., Bennett, D., Brown, B.L., 2016. P300 Amplitude in Alzheimer's Disease: A Meta-Analysis and Meta-Regression. Clin EEG Neurosci. 47, 48-55.

Horváth, A., Szűcs, A., Barcs, G., Noebels, J.L., Kamondi, A., 2016. Epileptic Seizures in Alzheimer Disease: A Review. Alzheimer Dis Assoc Disord. 30, 186-92.

Horváth, A., Szücs, A., Barcs, G., Kamondi, A., 2017. Sleep EEG Detects Epileptiform Activity in Alzheimer's Disease with High Sensitivity. J Alzheimers Dis. 56, 1175-1183.

Iaccarino, H.F., Singer, A.C., Martorell, A.J., Rudenko, A., Gao, F., Gillingham, T.Z., Mathys, H., Seo, J., Kritskiy, O, Abdurrob, F., Adaikkan, C., Canter, R.G., Rueda, R., Brown, E.N., Boyden, E.S., Tsai, L.H., 2016. Gamma frequency entrainment attenuates amyloid load and modifies microglia. Nature, 540, 230-235.

Iragui, V., Kutas, M., Salmon, D.P., 1996. Event-related brain potentials during semantic categorization in normal aging and senile dementia of the Alzheimer's type. Electroencephalog Clin Neurophysiol. 100, 392-406.

Jack, C.R., Bennett, D.A., Blennow, K., Carrillo, M.C., Dunn, B., Haeberlein S.B., Holtzman D.M., Jagust W., Jessen F., Karlawish J., Liu, E., Molinuevo, J.L., Montine, T., Phelps, C., Rankin, K.P., Rowe, C.C., Scheltens P, Siemers E, Snyder H.M., Sperling R. 2018. NIA-AA Research Framework: Toward a biological definition of Alzheimer's disease. Alzheimers Dement. 14,535-562.

Jelic, V., Johansson, S.E., Almkvist, O., Shigeta, M., Julin, P., Nordberg, A., Winblad, B., Wahlund, L.O., 2000. Quantitative electroencephalography in mild cognitive impairment: longitudinal changes and possible prediction of Alzheimer's disease. Neurobiol Aging. 21, 533-40. 
Jeong, J., Chae, J.H., Kim, S.Y., Han, S.H., 2001. Nonlinear dynamic analysis of the EEG in patients with Alzheimer's disease and vascular dementia. J Clin Neurophysiol. 18, 58-67.

Jeong, J., 2004. EEG dynamics in patients with Alzheimer's disease. Clin. Neurophysiol. 115, 1490-505.

Jiang, S., Qu, C., Wang, F., Liu, Y., Qiao, Z., Qiu, X., Yang, X., Yang Y., 2015. Using event-related potential P300 as an electrophysiological marker for differential diagnosis and to predict the progression of mild cognitive impairment: a meta-analysis. Neurol Sci. 36, 1105-12.

Kalweit, A.N., Yang, H., Colitti-Klausnitzer, J., Fülöp, L., Bozsó, Z., Penke, B., Manahan-Vaughan, D., 2015. Acute intracerebral treatment with amyloid-beta (1-42) alters the profile of neuronal oscillations that accompany LTP induction and results in impaired LTP in freely behaving rats. Front Behav Neurosci. 9:103.

Kar, S., Slowikowski.,S.P.M., Westaway, D., Mount, H.T.J., 2004. Interactions between $\beta$-amyloid and central cholinergic neurons: implications for Alzheimer's disease. J Psychiatry Neurosci. 29, 427-441.

Katada, E., Sato, K., Sawaki, A., Dohi, Y., Ueda, R., \& Ojika, K., 2003. Long-term effects of donepezil on P300 auditory event-related potentials in patients with Alzheimer's disease. J Geriatr Psychiatry Neurol. 16, 39-43.

Kutas, M., Federmeier, K.D., 2011. Thirty years and counting: finding meaning in the n400 component of the event-related brain potential (erp) Annu Rev Psychol. 62, 621-647.

Lai, C.L., Lin, R.T., Liou, L.M., Liu, C.K., 2010. The role of event-related potentials in cognitive decline in Alzheimer's disease. Clin Neurophysiol.121, 194-9.

Laursen, B., Mørk, A., Kristiansen, U., Bastlund, .JF. 2014. Hippocampal P3-like auditory event-related potentials are disrupted in a rat model of cholinergic degeneration in Alzheimer's disease: reversal by donepezil treatment. J Alzheimers Dis. 42, 1179-89.

Lam, A.D., Deck, G., Goldman, A., Eskandar, E.N., Noebels, J., Cole, A.J., 2017. Silent hippocampal seizures and spikes identified by foramen ovale electrodes in Alzheimer's disease. Nat Med. 23, 678-80.

Leiser, S.C., Dunlop, J., Bowlby, M.R., Devilbiss, D.M., 2011. Aligning strategies for using EEG as a surrogate biomarker: a review of preclinical and clinical research. Biochem Pharmacol. 81, 1408-21.

Lindín, M., Correa, K., Zurrón, M., Díaz, F., 2013. Mismatch negativity (MMN) amplitude as a biomarker of sensory memory deficit in amnestic mild cognitive impairment. Front Aging Neurosci. 5, 79.

López, M.E., Bruña, R., Aurtenetxe, S., Pineda-Pardo, J.Á., Marcos, A., Arrazola, J., Reinoso ,A.I., Montejo, P., Bajo, R., Maestú, F., 2014. Alpha-band hypersynchronization in progressive mild cognitive impairment: a magnetoencephalography study. J Neurosci. 34, 14551-9.

López, M.E., Cuesta, P., Garcés, P., Castellanos, P.N., Aurtenetxe, S., Bajo, R., Marcos, A, Delgado, M.L., Montejo, P., López-Pantoja, J.L., Maestú, F., Fernandez A., 2014. MEG spectral analysis in subtypes of mild cognitive impairment. 36, 1095-112.

López, M.E., Turrero, A., Cuesta, P., López-Sanz , D., Bruña, R., Marcos, A., Gil, P., Yus, M., Barabash, A., Cabranes, J.A., Maestú, F., Fernández, A. 2016. Searching for Primary Predictors of Conversion from Mild Cognitive Impairment to Alzheimer's Disease: A Multivariate Follow-Up Study. Journal of Alzheimer's Disease. 52, 133-43.

Luckhaus, C., Grass-Kapanke, B., Blaeser, I., Ihl, R., Supprian, T., Winterer, G., Zielasek, J., Brinkmeyer, J., 2008. Quantitative EEG in progressing vs stable mild cognitive impairment (MCI): results of a 1-year follow-up study. Int J Geriatr Psychiatry. 23, 1148-55.

Maestri, M., Carnicelli, L., Tognoni, G., Di Coscio, E., Giorgi, F.S., Volpi, L., Economou, N.T., Ktonas, P., Ferri, R., Bonuccelli, U., Bonanni, E., 2015. Non-rapid eye movement sleep instability in mild cognitive impairment: a pilot study. Sleep Med. 16, 1139-1145.

Maestú, F., Campo, P., Del Río, D., Moratti, S., Capilla, A,. Fernández, A., Gil, P., Ortiz, T., 2008. Increased biomagnetic activity in the ventral pathway in mild cognitive impairment. Clin Neurophysiol. 119, 1320-1327.

Maestú, F., Peña, J.M., Garcés, P., González, S., Bajo, R., Bagic, A., Cuesta, P., Funke, M., Mäkelä, J.P., Menasalvas, E., Nakamura, A., Parkkonen, L., López, M.E., Del Pozo, F., Sudre, G., Zamrini, E., Pekkonen, E., Henson, R.N., Becker, J.T, 2015. A multicenter study of the early detection of synaptic dysfunction in Mild Cognitive Impairment using Magnetoencephalography-derived functional connectivity. Neuroimage Clin. 9, 1039. 
Mertens, J., Paquola, A.C., Ku, M., Hatch, E., Böhnke, L., Ladjevardi, S., McGrath, S., Campbell, B., Lee, H., Herdy, J.R., Gonçalves, J.T., Toda, T., Kim, Y., Winkler, J., Yao, J., Hetzer, M.W., Gage, F.H., 2015. Directly Reprogrammed Human Neurons Retain Aging-Associated Transcriptomic Signatures and Reveal Age-Related Nucleocytoplasmic Defects. Cell Stem Cell. 17, 705-18.

Minkeviciene, R, Rheims, S, Dobszay, MB, Zilberter, M, Hartikainen, J, Fülöp, L, Penke, B, Zilberter, Y, Harkany ,T, Pitkänen, A, Tanila, H., 2009. Amyloid beta-induced neuronal hyperexcitability triggers progressive epilepsy. J Neurosci. 29, 3453-62.

Missonnier, P., Deiber, M.P., Gold, G., Herrmann, F.R., Millet, P., Michon, A., Fazio-Costa, L., Ibañez, V., Giannakopoulos, P., 2007. Working memory load-related electroencephalographic parameters can differentiate progressive from stable mild cognitive impairment. Neuroscience. 150, 346-56.

Morgan, C.D., Murphy, C., 2002. Olfactory event-related potentials in Alzheimer's disease. J Int Neuropsychol Soc. 8, 753-763

Morison, G., Tieges, Z., Kilborn, K., 2013. Analysis of Electroencephalography Activity in Early Stage Alzheimer's Disease Using a Multiscale Statistical Complexity Measure. Advanced Science Letters. 19, 24142418.

Mucke, L., Selkoe, D.J., 2012. Neurotoxicity of amyloid $\beta$-protein: synaptic and network dysfunction. Cold Spring Harb Perspect Med. 2, a006338.

Nakamura, A., Cuesta, P., Kato, T., Arahata, Y., Iwata, K., Yamagishi, M., Kuratsubo, I., Kato, K., Bundo, M., Diers, K., Fernández, A., Maestú, F., Ito, K., 2017. Early functional network alterations in asymptomatic elders at risk for Alzheimer's disease. Sci Reports. Sci Rep. 7, 6517.

Nunez, P.L., 2000. Toward a quantitative description of large-scale neocortical dynamic function and EEG. Behav Brain Sci. 23, 371-398.

Nunez, P.L., Srinivasan R., 2006. Electric Fields of the Brain: The Neurophysics of EEG, 2nd edition, Oxford University Press.

Nunez, P.L., Srinivasan R., Fields, R.D., 2015. EEG functional connectivity, axon delays and white matter disease, Clinical Neurophysiology. 126, 110-120.

Oakley, H., Cole, SL., Logan, S., Maus, E., Shao, P., Craft, J., Guillozet-Bongaarts, A., Ohno, M., Disterhoft, J., Van Eldik, L., Berry, R., Vassar, R., 2016. Intraneuronal beta-amyloid aggregates, neurodegeneration, and neuron loss in transgenic mice with five familial Alzheimer's disease mutations: potential factors in amyloid plaque formation. J Neurosci. 26, 10129-40.

Olichney, J.M., Morris, S.K., Ochoa, C., Salmon, D.P., Thal, LJ., Kutas, M., Iragui, V.J., 2002a. Abnormal verbal event related potentials in mild cognitive impairment and incipient Alzheimer's disease. J Neurol Neurosurg Psychiatry. 73, 377-84.

Olichney, J.M., Riggins, B.R., Morris, S.K., Salmon, D.P., Kutas, M., Iragui, V.J., 2002b. Reduced effects of word repetition on the $\mathrm{n} 400$ and lpc event-related potentials are common in mild Alzheimer's disease and mild cognitive impairment converters. Neurology. 58, A216.

Olichney J.M., Iragui, V.J., Salmon, D.P., Riggins, B.R., Morris, S.K., Kutas M., 2006. Absent event-related potential (ERP) word repetition effects in mild Alzheimer's disease. Clin Neurophysiol. 117, 1319-30.

Olichney, J.M., Yang J., Taylor J., Kutas M., 2011. Cognitive Event-Related Potentials: Biomarkers of Synaptic Dysfunction Across the Stages of Alzheimer's Disease. Journal of Alzheimer's Disease. 26, 215-228.

Olichney J.M., Pak, J., Salmon, D.P., Yang, J.C., Gahagan, T., Nowacki, R., Hansen, L., Galasko, D., Kutas, M., Iragui-Madoz, V.J., 2013. Abnormal P600 word repetition effect in elderly persons with preclinical Alzheimer's disease. Cogn Neurosci. 4, 143-51.

Onofrj, M., Thomas, A., Iacono, D., Luciano, A.L., Di Iorio A., 2003. The effects of a cholinesterase inhibitor are prominent in patients with fluctuating cognition: a part 3 study of the main mechanism of cholinesterase inhibitors in dementia. Clin Neuropharmacol. 26, 239-51.

Onos, K.D., Sukoff Rizzo, S.J., Howell, G.R., Sasner M., 2016. Toward more predictive genetic mouse models of Alzheimer's disease. Brain Res Bull. 122, 1-11.

Palop, J.J., Chin, J., Roberson, E.D., Wang, J., Thwin ,M.T., Bien-Ly, N., Yoo, J., Ho, KO., Yu, GQ., Kreitzer, A., Finkbeiner, S., Noebel,s J.L., Mucke L., 2007. Aberrant excitatory neuronal activity and compensatory remodeling of inhibitory hippocampal circuits in mouse models of Alzheimer's disease. Neuron. 55, 697-711. 
Palop, J.J., Mucke, L., 2016. Network abnormalities and interneuron dysfunction in Alzheimer disease. Nat Rev Neurosci. 17, 777-792.

Papaliagkas, V.T., Anogianakis, G., Tsolaki, M.N., Koliakos, G., Kimiskidis,V.K., 2009. Prediction of conversion from mild cognitive impairment to Alzheimer's disease by CSF cytochrome c levels and N200 latency. Curr Alzheimer Res. 6, 279-84.

Papaliagkas, V.T., Kimiskidis, V.K., Tsolaki, M.N., Anogianakis, G., 2011. Cognitive event-related potentials: longitudinal changes in mild cognitive impairment. Clin Neurophysiol. 122, 1322-6.

Papazoglou, A., Soos, J., Lundt, A., Wormuth, C., Ginde, V.R. Müller, R., Henseler, C., Broich, K., Xie, K., Haenisch, B., Ehninger, D., Weiergräber, M., 2017. Motor Cortex Theta and Gamma Architecture in Young Adult APPswePS1dE9 Alzheimer Mice. PLoS One. 12, e0169654.

Parrino, L., Ferri, R., Bruni, O., Terzano, M.G., 2012. Cyclic alternating pattern (CAP): the marker of sleep instability. Sleep Med Rev. 16, 27-45.

Peeraer, E., Bottelbergs, A., Van Kolen, K., Stancu, I.C., Vasconcelos, B., Mahieu, M., Duytschaever, H., Ver Donck, L., Torremans, A., Sluydts, E., Van Acker, N., Kemp, JA., Mercken, M., Brunden, K.R., Trojanowski, J.Q., Dewachter, I., Lee V.M., Moechars, D., 2015. Intracerebral injection of preformed synthetic tau fibrils initiates widespread tauopathy and neuronal loss in the brains of tau transgenic mice. Neurobiol Dis. 73, 83-95.

Polich, J., Corey-bloom, J., 2005. Alzheimer's disease and P300: Review and evaluation of task and modality. Curr Alzheimer Res. 300, 515-525.

Polikar, R., Topalis, A., Green, D., Kounios, J., Clark C.M., 2007. Comparative multiresolution wavelet analysis of ERP spectral bands using an ensemble of classifiers approach for early diagnosis of Alzheimer's disease. Comput Biol Med. 37, 542-58.

Quiroz, Y.T., Ally, B.A., Celone, K., McKeever, J., Ruiz-Rizzo, A.L., Lopera, F., Stern, C.E., Budson, A.E. Event-related potential markers of brain changes in preclinical familial Alzheimer disease. Neurology. 77(5):46975 .

Rammes, G., Hasenjäger, A., Sroka-Saidi, K., Deussing, J.M., Parsons, C.G., 2011. Therapeutic significance of NR2B-containing NMDA receptors and mGluR5 metabotropic glutamate receptors in mediating the synaptotoxic effects of beta-amyloid oligomers on long-term potentiation (LTP) in murine hippocampal slices. Neuropharmacology. 60, 982-90.

Rong, X., 2016. Levetiracetam might act as an efficacious drug to attenuate cognitive deficits of Alzheimer's disease. Curr Topics Medicinal Chem. 16, 565-573.

Rossini, P.M., Del Percio, C., Pasqualetti, P., Cassetta, E., Binetti, G., Dal Forno, G., Ferreri, F., Frisoni, G., Chiovenda, P., Miniussi, C., Parisi, L., Tombini, M., Vecchio, F., Babiloni C., 2006. Conversion from mild cognitive impairment to Alzheimer's disease is predicted by sources and coherence of brain electroencephalography rhythms. Neuroscience. 143, 793-803.

Rugg, M.D., Curran, T., 2007 Event-related potentials and recognition memory. Trends Cogn Sci. 11, 251-7.

Scarmeas, N., Honig, L.S., Choi, H., Cantero, J., Brandt J., Blacker, D., Albert, M., Amatniek, J.C., Marder, K., Bell, K., Hauser, W.A., Stern Y., 2009. Seizures in Alzheimer disease: who, when, and how common? Arch Neurol. 66, 992-7.

Scott L., Kiss, T., Kawabe, T.T., Hajós, M., 2016. Neuronal network activity in the hippocampus of tau transgenic (Tg4510) mice. Neurobiol Aging. 37, 66-73.

Shankar, G.M., Li, S., Mehta, T.H., Garcia-Munoz, A., Shepardson, NE.., Smith, I., Brett, F.M., Farrell, M.A., Rowan, M.J., Lemere, C.A., Regan, C.M., Walsh, D.M., Sabatini, B.L., Selkoe, D.J., 2008. Amyloid-beta protein dimers isolated directly from Alzheimer's brains impair synaptic plasticity and memory. Nat Med. 14, 837-42.

Siwek ,M.E., Müller, R., Henseler, C., Trog, A., Lundt, A., Wormuth, C., Broich, K., Ehninger, D., Weiergräber, M., Papazoglou A., 2015. Altered theta oscillations and aberrant cortical excitatory activity in the 5XFAD model of Alzheimer's disease. 2015, 781731.

Smith, K., Escudero, J., 2017. The complex hierarchical topology of EEG functional connectivity. J Neurosci Methods. 276, 1-12.

Stam, C.J., Nolte, G., Daffertshofer, A., 2007. Phase lag index: assessment of functional connectivity from multi channel EEG and MEG with diminished bias from common sources. Hum Brain Mapp. 28, 1178-93. 
Stoiljkovic, M., Kelley, C., Stutz, B., Horvath, T.L., Hajós, M., 2018. Altered Cortical and Hippocampal Excitability in TgF344-AD Rats Modeling Alzheimer's Disease Pathology. Cereb Cortex. 29: 2716-2727.

Subota, A, Pham, T., Jetté, N., Sauro, K., Lorenzetti, D., Holroyd-Leduc, J., 2017. The association between dementia and epilepsy: A systematic review and meta-analysis. Epilepsia. 58, 962-972.

Tabar ,V., Studer, L., 2014. Pluripotent stem cells in regenerative medicine: challenges and recent progress. Nat Rev Genet. 15, 82-92.

Takahashi, T., Tanabe, K., Ohnuki, M., Narita, M., Ichisaka, T., Tomoda, K., Yamanaka, S., 2007 Induction of Pluripotent Stem Cells from Adult Human Fibroblasts by Defined Factors. Cell. 5, 861-872

Takahashi, T., 2013. Complexity of spontaneous brain activity in mental disorders. Prog Neuropsychopharmacol Biol Psychiatry. 45, 258-66.

Tamagnini, F., Novelia, J., Kerrigan, T.L., Brown, J.T., Tsaneva-Atanasova, K., Randall, A.D., 2015. Altered intrinsic excitability of hippocampal CA1 pyramidal neurons in aged PDAPP mice. Front Cell Neurosci. 9, 372.

van Diessen, E., Numan, T., van Dellen, E., van der Kooi, AW., Boersma, M., Hofman, D., van Lutterveld, R., van Dijk,BW., van Straaten, EC., Hillebrand, A., Stam, CJ., 2015. Opportunities and methodological challenges in EEG and MEG resting state functional brain network research. Clin Neurophysiol. 126, 1468-81.

Varela, F., Lachaux, JP., Rodriguez, E., Martinerie, J., 2001. The brainweb: phase synchronization and largescale integration. Nat Rev Neurosci. 2, 229-39.

van Straaten, E.C.W., Stam, C.J., 2013. Structure out of chaos: Functional brain network analysis with EEG, MEG, and functional MRI. Eur Neuropsychopharmacol. 23, 7-18.

Varga, A.W., Wohlleber, M.E., Gimenez, S., Romero, S., Alonso, J.F., Ducca, E.L., Kam, K., Lewis, C., Tanzi, E.B., Tweardy, S., Kishi, A., Parekh, A., Fischer, E., Gumb, T., Alcolea, D., Fortea, J., Lleo, A., Blennow, K., Zetterberg, H., Mosconi, L., Glodzik, L., Pirraglia, E., Burschtin, O.E., de Leon, M.J., Rapoport, D.M., Lu, S.E., Ayappa, I., Osorio, RS., 2016. Reduced Slow-Wave Sleep Is Associated with High Cerebrospinal Fluid Abeta42 Levels in Cognitively Normal Elderly. Sleep. 39, 2041-2048.

Vecchio, F., Miraglia, F., Piludu, F., Granata, G., Romanello, R., Caulo, M., Onofrj, V., Bramanti, P., Colosimo, C., Rossini, P.M., 2017. "Small World" architecture in brain connectivity and hippocampal volume in Alzheimer's disease: a study via graph theory from EEG data. Brain Imaging Behav. 11, 473-485.

Villegas-Llerena, C., Phillips ,A, Garcia-Reitboeck, P., Hardy, J., Pocock, J.M., 2016. Microglial genes regulating neuroinflammation in the progression of Alzheimer's disease. Curr Opin Neurobiol. 36, 74-81.

Voytek, B., Knight, R.T., 2015. Dynamic network communication as a unifying neural basis for cognition, development, aging, and disease. Biol Psychiatry. 77, 1089-97.

Vossel, K.A., Beagle, A.J., Rabinovici, G.D., Shu, H., Lee, S.E., Naasan, G., Hegde, M., Cornes, S.B., Henry, M.L., Nelson, A.B., Seeley, W.W., Geschwind, M.D., Gorno-Tempini, M.L., Shih, T., Kirsch, H.E., Garcia, P.A., Miller, B.L, Mucke L., 2013. Seizures and epileptiform activity in the early stages of Alzheimer disease. JAMA Neurol. 70, 1158-66.

Vossel, K.A., Ranasinghe, K.G., Beagle, A.J., Mizuiri, D., Honma, S.M., Dowling, A.F., Darwish, S.M., Van Berlo, V., Barnes, D.E., Mantle, M., Karydas, A.M., Coppola, G., Roberson, E.D., Miller, B.L., Garcia, P.A., Kirsch, H.E., Mucke, L., Nagarajan, S.S., 2016. Incidence and impact of subclinical epileptiform activity in Alzheimer's disease. Ann Neurol. 80, 858-870.

Walsh, C., Drinkenburg, W.H., Ahnaou, A., 2017. Neurophysiological assessment of neural network plasticity and connectivity: Progress towards early functional biomarkers for disease interception therapies in Alzheimer's disease. Neurosci Biobehav Rev. 73, 340-358.

Xu, H., Gelyana, E., Rajsombath, M., Yang, T., Li, S., Selkoe, D., 2016. Environmental Enrichment Potently Prevents Microglia-Mediated Neuroinflammation by Human Amyloid beta-Protein Oligomers. J Neurosci. 36, 9041-56.

Yamada, K., Holth, J.K., Liao, F., Stewart, F.R., Mahan, T.E., Jiang, H., Cirrito, J.R., Patel, T.K., Hochgräfe, K., Mandelkow, E.M., Holtzman, D.M., 2014. Neuronal activity regulates extracellular tau in vivo. J Exp Med. 211, 387-93.

Yener, G.G., Guntekin, B., Oniz, A., Basar, E., 2007. Increased frontal phase-locking of event-related theta oscillations in Alzheimer patients treated with cholinesterase inhibitors. Int J Psychophysiol. 64, 46-52. 
Yener, G.G., Başar, E., 2013. Biomarkers in Alzheimer's disease with a special emphasis on event-related oscillatory responses. Suppl Clin Neurophysiol. 62, 237-73.

Yulug, B., Hanoglu, L., Kilic, E., 2017. Does sleep disturbance affect the amyloid clearance mechanisms in alzheimer's disease? Psychiatry Clin Neurosci. 71, 673-677.

Zhang, X., Zhong, W., Brankačk, J., Weyer, S.W., Müller, U.C., Tort, A.B., Draguhn, A., 2016. Impaired thetagamma coupling in APP-deficient mice. Sci Rep. 6, 21948.

Ziyatdinova, S., Gurevicius, K., Kutchiashvili, N., Bolkvadze, T., Nissinen, J., Tanila, H., Pitkänen A. 2011. Spontaneous epileptiform discharges in a mouse model of Alzheimer's disease are suppressed by antiepileptic drugs that block sodium channels. Epilepsy Res. 94, 75-85. 


\section{Panel 1: Dimensions of electrophysiological signals}

\section{Determinism - Randomness}

- Electrophysiological signals are deterministic when defined inputs to and parameters of neural sources of electrophysiological signals produce scalp rsEEG signals always showing the same characteristics.

- Local field potentials (LFPs) and ongoing electroencephalographic (EEG) signals do not typically reflect a pure determinism.

\section{Simplicity - Complexity}

- Electrophysiological signals are generated by simple interactions into a neural system when they are predictable as features (i.e. electrophysiological signals recorded during motor reflexes or long-term potentiation).

- These signals are generated by complex interactions into a neural system when some nodes of the network are unknown and electrophysiological signals are not predictable. LFPs and ongoing EEG signals do not typically reflect simple interactions.

\section{Linearity - Nonlinearity}

- Electrophysiological signals are linear when any linear combination of inputs to neural system generating those signals produces the same linear combination of them, each of which would have been produced in isolation by a given input. When that neural system operates as a random linear system, amplitudes and phases of each electrophysiological signal are expected to be independent of each other.

- When electrophysiological signals are generated by a random nonlinear system, their amplitudes and frequencies may show harmonic relationships in the bispectrum and bicoherence. LFPs and ongoing EEG signals can show some nonlinearity in patients with epileptic seizures or several mental disorders.

\section{Stationarity - Nonstationarity}

- Electrophysiological signals are stationary when they show constant statistical features (e.g., mean, variance, and autocorrelation function, power spectra) over time.

- The condition of stationarity or "quasi-stationary" can be found in electrophysiological signals for relatively short measurement periods of a few seconds.

\section{Phase - Nonphase locking}

- Electrophysiological signals are phase-locked when external (sensory stimuli) or endogenous (cognitive operations, motor acts) events change their phase.

- The same event can parallelly evoke phase-locked and nonphase-locked changes in electrophysiological signals. 
Panel 2: Experimental conditions of recordings of electroencephalographic (EEG) and magnetoencephalographic (MEG) signals in humans

\section{Resting state and sleep}

- EEG/MEG signals are recorded for few minutes during eyes-closed and -open conditions in quiet wakefulness to monitor brain vigilance systems or during the night to probe neurophysiological oscillations underpinning sleep.

- $\quad$ EEG/MEG spectral analysis. Comparison of EEG spectral markers in relation to AD status and progression (control subjects as a reference).

- Evaluation of slow waves and signs of overexcitability and epileptiform activity (e.g., spikes, waves, spike-waves).

\section{Evoked and Event-Related Potentials/Magnetic fields}

- $\quad$ EEG/MEG signals are recorded for some tens of minutes during repeated sensory stimuli (e.g., visual, auditory, etc.) with no response required in paradigms of evoked potentials (EPs) or magnetic fields (EMFs);

- Those signals are recorded for some minutes during repeated sensory stimuli (e.g., visual, auditory, etc.) and some cognitive information processing and motor response in paradigms of event-related potentials (ERPs) or magnetic fields (ERMFs);

- $\quad$ EEG/MEG signals associated with any single stimulus are averaged to produce ERPs/ERMFs, analyzed in the time domain as amplitude and latency of negative and positive peaks.

\section{Evoked and Event-Related Oscillations}

- $\quad$ Evoked/event-related oscillations (EROs) or their magnetic counterpart (event-related magnetic oscillations, ERMOs) probe the event-related and phase-locked changes of EEG (MEG) rhythms in the frequency domain.

- EROs-ERMOs are obtained by (1) the FFT-based passband filtering of ERPs (ERMFs) accompanying rare stimuli. For this passband filtering, standard frequency bands are used, namely delta $(<4 \mathrm{~Hz})$, theta $(4-7 \mathrm{~Hz})$, alpha $(8-12 \mathrm{~Hz})$, beta $(13-30 \mathrm{~Hz})$, and gamma $(30-45 \mathrm{~Hz})$; and (2) the measurement of the maximum amplitude from positive to negative peaks of the filtered ERPs at any electrode.

- Event-related desynchronization/synchronization (ERD/ERS) of ongoing EEG (MEG) rhythms probe nonphase-locked reduction of alpha and beta power (ERD) as well as the increase of theta and gamma power (ERS) as signs of cortical activation. 


\section{Mismatch negativity (MMN) and oddball}

- In the pre-attentive MMN paradigm, EEG/MEG signals are recorded for some tens of minutes during repeated sensory stimuli (e.g., visual, auditory, etc.) with no response required while the subject is focused on another task (e.g. reading or watching videos); these stimuli are frequent (about $80 \%$ ) or rare $(20 \%)$ and presented in (pseudo)random order.

- $\quad$ EEG/MEG signals associated with any single stimulus are averaged to produce ERPs/ERMFs and analyzed in the time domain as amplitude and latency of a frontal negative component peaking at about $200 \mathrm{~ms}$ post-stimulus, the MMN. Those signals may be also analyzed in the frequency domain in the standard bands from delta to gamma.

- In the attentive oddball paradigm, EEG/MEG signals are recorded for some tens of minutes during repeated sensory stimuli (e.g., visual, auditory, etc.); these stimuli are frequent (about $80 \%$ ) or rare $(20 \%)$, presented in (pseudo)random order and the subject has to respond only to the rare "target" stimuli (e.g. via counting or button press).

- $\quad$ EEG/MEG signals associated with any single stimulus are averaged to produce ERPs/ERMFs and analyzed in the time domain mainly as amplitude and latency of a posterior positive component peaking at about $300 \mathrm{~ms}$ post-stimulus, the P300 or P3b. Those signals may be also analyzed in frequency domain in the standard bands from delta to gamma.

\section{N400/syntactic P600}

- In the semantic-incongruence N400 paradigm, EEG/MEG signals are recorded for some tens of minutes during couples of linguistic stimuli (e.g., visual, auditory, etc.) with no response required while the subject is focused on processing them; in some trials, the second stimulus is selected to produce an incongruity effect at either the semantic or phonological level.

- EEG/MEG signals associated with the couples of stimuli are averaged to produce ERPs/ERMFs and analyzed in the time domain as amplitude and latency of negative peaks. The effect of semantic incongruence modulates a temporoparietal negative ERP peak at about $+400 \mathrm{~ms}$ poststimulus, the N400. Regardless of incongruence, a distinct mid-frontal N400 (FN400) is related instead to the repetition effect and familiarity-related memory processes. Hearing or reading grammatical errors and other syntactic anomalies also induces a modulation of a centro-parietal "syntactic" P600.

- $\quad$ EEG/MEG signals associated with the couples of stimuli may be analyzed in the frequency domain in the standard bands from delta to gamma.

\section{Memory P600}

- In the episodic-memory P600 paradigm, EEG/MEG signals are recorded for some tens of minutes during sensory stimuli (e.g., words) during both a first encoding condition and, after a pause (usually between 1 minute and 1 hour), a second retrieval (recall or recognition) condition while the subject is focused on the stimuli. In the two conditions, the words produce an effect related to memory encoding or retrieval. In the retrieval condition, the subject usually responds with a "seen before" or "unseen before" decision.

- EEG/MEG signals associated with the stimuli are averaged to produce ERPs/ERMFs and analyzed in the time domain as amplitude and latency of a late positive peak. In the retrieval condition, the effect of successful recognition modulates a positive (left) parietal ERP peak at about $+600 \mathrm{~ms}$ poststimulus, the P600.

- $\quad$ EEG/MEG signals associated with the stimuli may be analyzed in the frequency domain at the standard bands from delta to gamma. 
Panel 4: Experimental conditions of recordings of local field potentials (LFPs) and electroencephalographic (EEG) signals in rodents

\section{Freely behaving}

- LFP/EEG signals are recorded for 24 hours (12 hours with light on and 12 hours of light off) to several days to monitor wake-sleep cycle, evolution of LFP/EEG frequency bands from 1 - > $100 \mathrm{~Hz}$ and behavioral activity and sleep structure over the day.

- $\quad$ EEG spectral analysis (FFT, wavelet) combined with simultaneous video, accelerometer or electromyographic (EMG) recording. Segmentation of the recording into movement, waking immobility or more detailed sleep states. Comparison of EEG spectral markers in different behavioral states. Localization of LFP sources by silicon-based linear probes (Csicsvari et al., 2003) or distributed horizontal cortical electrode arrays (Lundt et al., 2016). Subcortical-cortical connectivity and the role of interneuron dysfunction (Gurevicius et al., 2013, Ahnaou et al., 2014). Combination of EEG or LFPs with in vivo calcium imaging (Busche et al.,2015) or optogenetics (Iaccarino et al., 2016).

- Evaluation of overexcitability, epileptiform activity (e.g., spikes, waves, spike-waves) and epileptic seizures during wake-sleep cycle.

\section{Sensory and cognitive-motor processing}

- $\quad$ LFP/EEG signals are recorded for some minutes during repeated sensory (e.g., visual, auditory, etc.) stimuli with no response required in paradigms of evoked potentials (EPs);

- $\quad$ LFP/EEG signals are recorded for some minutes during repeated sensory (e.g., visual, auditory, etc.) stimuli and some cognitive information processing and motor response are required in paradigms of event-related potentials (ERPs);

- LFP/EEG signals associated with any single stimulus are averaged to produce ERPs or analyzed in the frequency domain to produce spectral markers at frequency bands of interest from 1 to $>100 \mathrm{~Hz}$. Spectral markers include the computation of coherence or other indexes of functional coupling of LFP/EEG signals at electrode pairs.

\section{Long term potentiation (LTP) and depression (LTD) in anesthesia}

- $\quad$ LFP and single neuron signals are recorded from hippocampus during LTP/LTD paradigms in the full complexity of an intact brain. Such recordings are traditionally performed under general anesthesia to exclude movement and vigilance-related confounding factors.

- Brainstem or hippocampal electrical stimulation to induce LTP and enhanced theta (4-8 Hz) oscillations in the hippocampus. 
Figure 1. Schematic overview of the scale of spatial and temporal resolution of measurement methods used for electrophysiology and functional neuroimaging. Measurement methods are electroencephalography (EEG), magnetoencephalography (MEG), near-infrared spectroscopy (NIRS), functional magnetic resonance imaging (fMRI), electrocorticography (ECOG), local field potential (LFP) recordings, micro-electrode array (MEA) recordings, and microelectrode (ME) recordings. Non-invasive methods are shown in blue and invasive methods are shown in red. Adapted from van Gerven et al., 2009, J Neural Eng. 2009 (courtesy from the Publisher).

Figure 2. Neural synchrony as a multiscale phenomenon. A | Local scale: within a small brain region or local network, at least three levels of analysis can be distinguished. a Synchrony between single units in monkey area $V 1$ stimulated by a drifting grating, as measured by a cross-correlogram. $b \mid$ Local field potentials (LFPs) from eight recording electrodes in the suprasylvian gyrus of an awake cat. Maximum separation between electrodes was $7 \mathrm{~mm}$. The overlapping traces show a brief episode of synchronization between the fast oscillations. $c \mid$ Transient episodes of synchrony within a population of neurons recorded intracranially over the occipitotemporal junction in an epileptic patient performing a visual discrimination task. Time-frequency analysis revealed an enhancement of the local energy in the gamma band around 300 ms following the visual stimulation. This enhancement corresponds to the transient synchronization of underlying populations. $d \mid$ When recorded from a surface electrode, such synchronous patches appear as spatial summation of cortical responses that give rise to transient increases in the gamma band. $B \mid$ Large scale: patches of local synchrony in distant brain sites can enter synchrony during cognitive tasks. Synchronous patterns between distant scalp electrodes were recorded in normal subjects engaged in a face recognition task. Black lines link electrodes that are synchronous during the perception of the face. Adapted from Varela et al., Nat Rev Neurosci. 2001 (courtesy from the Publisher).

Figure 3. Putative neurophysiological models for the generation of "induced" nonphase-locked (ERD/ERS) and "evoked" phase-locked (ERP) changes in EEG (MEG) rhythms at the level of cortical pyramidal neurons in relation to a given event (i.e. stimulus, cognitive process, movement, etc.). Note that both circuits (i.e. "induced" and "evoked") are simultaneously active during the event and involve neurons of sensory organs, subcortical motor systems, activating ascending reticular system from brain stem, thalamocortical relay neurons (TCR), reticular thalamic nucleus (RE) neurons, and pyramidal neurons in the cerebral cortex. In these models, the nonphase-locked "induced" component of the EEG (MEG) signal might be due to prominent intrinsic thalamocortical and corticothalamic interactions modulated by input signals. In contrast, the phase-locked "evoked" component of the EEG (MEG) signal might be due to prominent inputs to thalamus and cerebral cortex. EEG $(M E G)$ is generated by the spatial and temporal (synchronization) summation of the post-synaptic potentials in cortical pyramidal neurons. Adapted from Pfurtscheller and Lopes da Silva, Electroencephalography and Clinical Neurophysiology, 1999 (courtesy from the Publisher). 
Figure 1

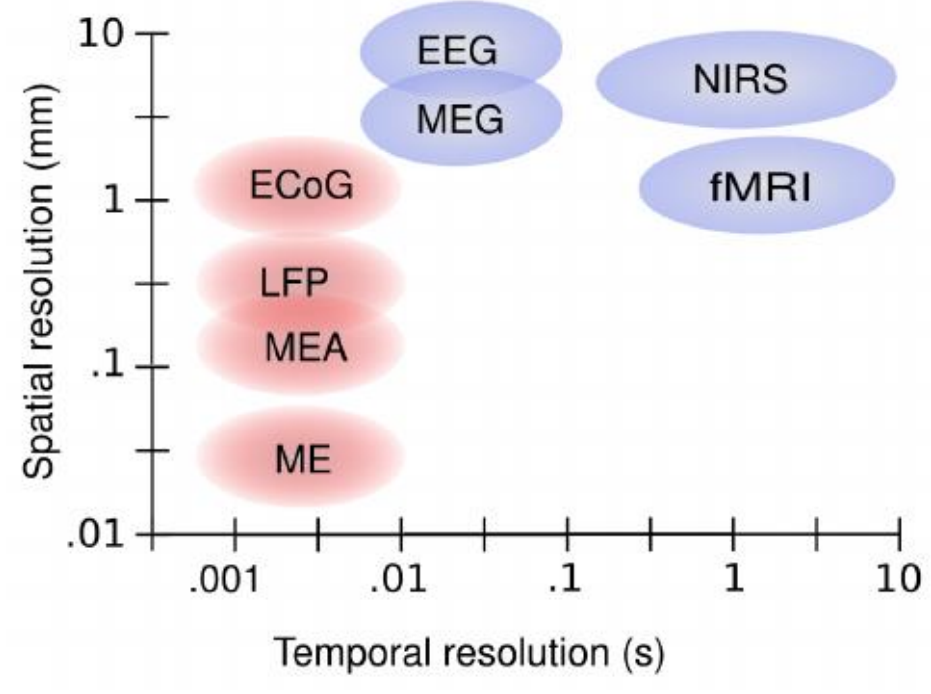


Figure 2

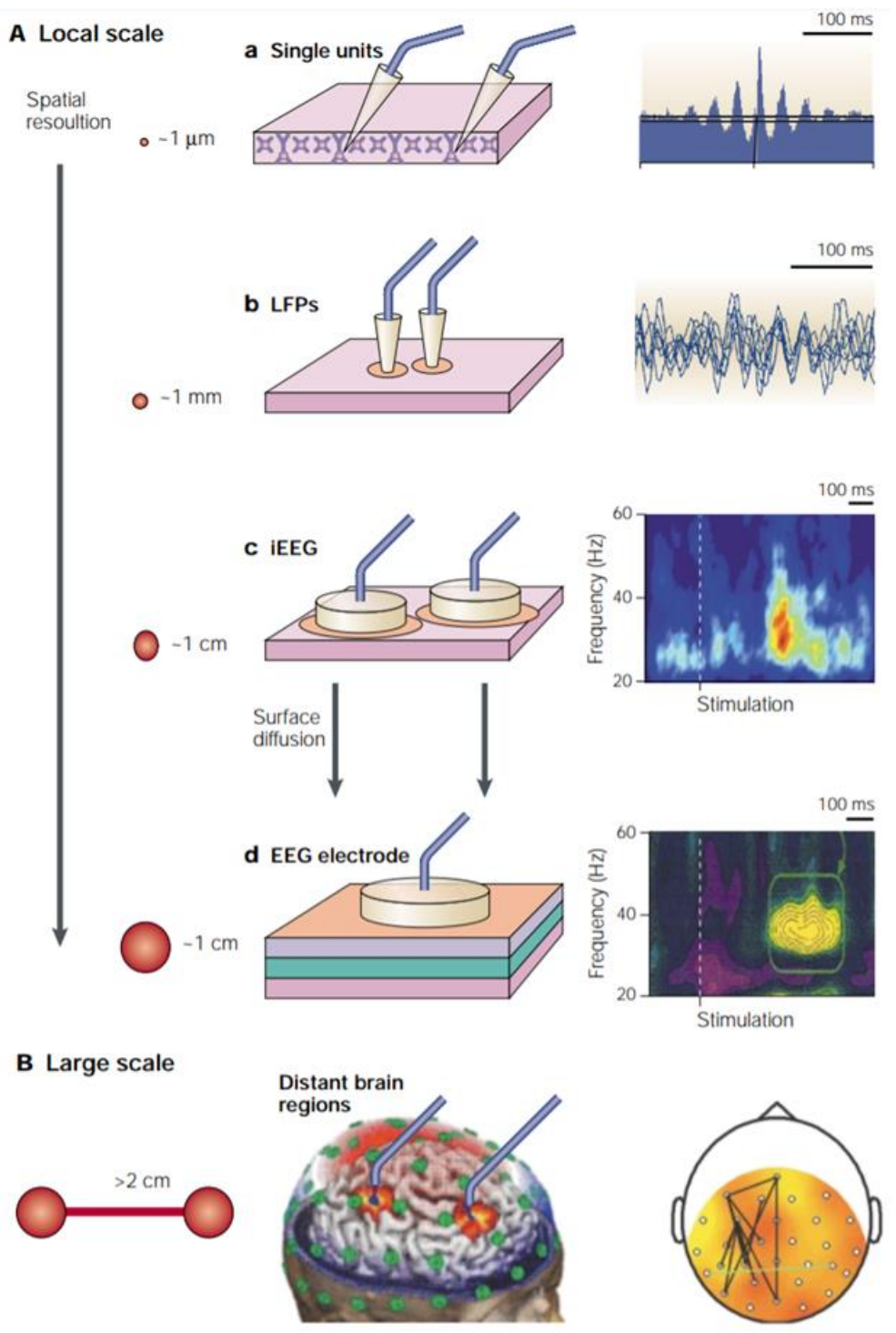


Figure 3

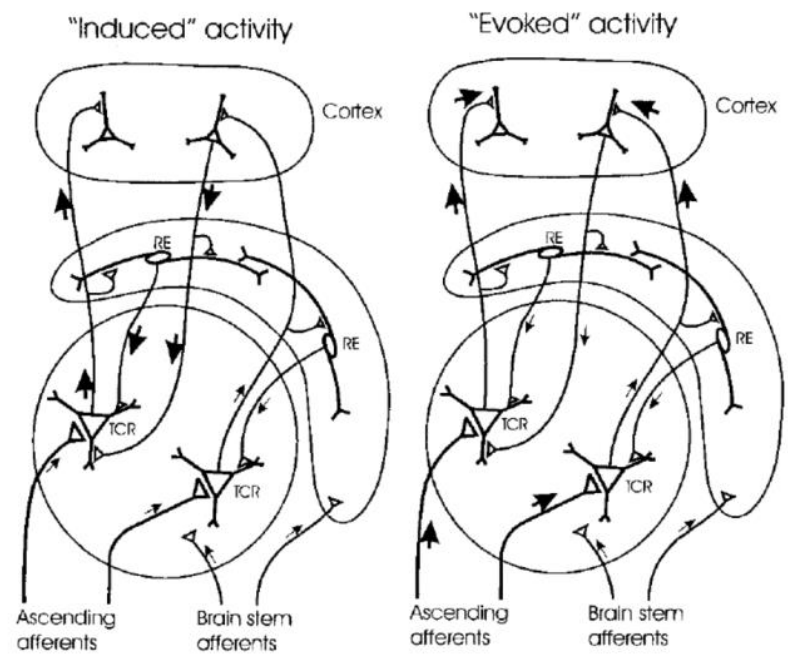

